\title{
Educational interventions to improve prescription and dispensing of antibiotics: a systematic review
}

\author{
Fátima Roque ${ }^{1,2,3}$, Maria Teresa Herdeiro ${ }^{2,4^{*}}$, Sara Soares ${ }^{2}$, António Teixeira Rodrigues ${ }^{2}$, Luiza Breitenfeld ${ }^{1}$ and \\ Adolfo Figueiras ${ }^{5}$
}

\begin{abstract}
Background: Excessive and inappropriate antibiotic use contributes to growing antibiotic resistance, an important public-health problem. Strategies must be developed to improve antibiotic-prescribing. Our purpose is to review of educational programs aimed at improving antibiotic-prescribing by physicians and/or antibiotic-dispensing by pharmacists, in both primary-care and hospital settings.
\end{abstract}

Methods: We conducted a critical systematic search and review of the relevant literature on educational programs aimed at improving antibiotic prescribing and dispensing practice in primary-care and hospital settings, published in January 2001 through December 2011.

Results: We identified 78 studies for analysis, 47 in primary-care and 31 in hospital settings. The studies differed widely in design but mostly reported positive results. Outcomes measured in the reviewed studies were adherence to guidelines, total of antibiotics prescribed, or both, attitudes and behavior related to antibiotic prescribing and quality of pharmacy practice related to antibiotics. Twenty-nine studies (62\%) in primary care and twenty-four (78\%) in hospital setting reported positive results for all measured outcomes; fourteen studies (30\%) in primary care and six (20\%) in hospital setting reported positive results for some outcomes and results that were not statistically influenced by the intervention for others; only four studies in primary care and one study in hospital setting failed to report significant post-intervention improvements for all outcomes. Improvement in adherence to guidelines and decrease of total of antibiotics prescribed, after educational interventions, were observed, respectively, in $46 \%$ and $41 \%$ of all the reviewed studies. Changes in behaviour related to antibiotic-prescribing and improvement in quality of pharmacy practice was observed, respectively, in four studies and one study respectively.

Conclusion: The results show that antibiotic use could be improved by educational interventions, being mostly used multifaceted interventions.

Keywords: Drug resistance microbial, Review, Behavior change, Education medical continuing, Education pharmacy continuing

\section{Background}

Antibiotic resistance is an important public-health issue, which is aggravated by the lack of new antimicrobial agents [1,2]. Inappropriate use of antibiotics is the main factor underlying microbial resistance [3,4]. Ecological studies in Europe suggest that there is a clear association

\footnotetext{
* Correspondence: teresaherdeiro@ua.pt

${ }^{2}$ Centre for Cell Biology, University of Aveiro (Centro de Biologia Celular CBC/UA); Campus Universitário de Santiago, 3810-193 Aveiro, Portugal ${ }^{4}$ CESPU, Instituto de Investigação e Formação Avançada em Ciências e Tecnologias da Saúde, Rua Central de Gandra, 1317, 4585-116 Gandra, PRD, Portugal

Full list of author information is available at the end of the article
}

between extent of antibiotic use and rate of resistance [5]. Excessive and inappropriate use of antibiotics is attributed to misprescription and to self-medication with "leftovers" from previous courses or with antibiotics dispensed in pharmacies without prescription [6,7]. In countries with a high incidence of self-medication with antibiotics, prescription of antibiotics is also high [7], suggesting that both practices are subject to the same cultural factors [8]. Physicians and pharmacists are the health professionals who exert most influence on patients' medication-related behavior. Many educational interventions to improve antibiotic-prescribing and/or 
dispensing have targeted those health professionals. Previous systematic reviews of the topic include Steinman's [9], which covered reports published prior to 2004 and on interventions directed at physicians. Other more recent reviews [10-13] have targeted specific areas, namely, respiratory tract infections [10,13], critical care [11], and acute care [12]. Therefore, there has been no general reviews, of the topic, including interventions on physicians a pharmacists to improve antibiotic prescription and dispensing. To close this gap, we carried out a critical review of educational programs aimed at improving antibioticprescribing by physicians and/or antibiotic-dispensing by pharmacists, in both primary-care and hospital settings.

\section{Methods}

\section{Literature search methodology}

For review purposes, we conducted a search of the MEDLINE-PubMED scientific database from January 2001 through December 2011. In addition, other papers were located by manual searches targeting journals, particularly those less likely to be indexed, and references cited by papers retrieved.

The search strategy was designed to identify relevant studies addressing antibiotic resistance and the prescribing/dispensing habits of health care providers (physicians and pharmacists) pre- and post-educational interventions. The following search terms and their equivalents were used in PubMed: ("intervention" OR "program" OR "health promotion" OR "education") AND ("pharmacists" OR "pharmacy" OR "physician” OR "health professionals" OR "clinician" OR "clinic" OR "practitioner" OR "general practitioner" OR "doctor") AND ("antibiotics" OR "antimicrobial”).

Based on previous reviews [14-17], we apply this selection criteria: (i) language: papers had to be published in English, French, Spanish or Portuguese; (ii) type of intervention: studies had to describe educational interventions; (iii) target population: educational interventions had to target physicians (general practitioners and all specialties) and/or pharmacists (population studies were included only if they also included interventions on pharmacists and/or physicians); and (iv) outcome measures: studies had to measure the effect of educational interventions on the prescribing behavior of physicians and/or dispensing behavior of pharmacists. Insofar as study design was concerned, no inclusion or exclusion criteria were stipulated because our aim was to use quality methodology to conduct a critical review of all published studies.

\section{Data-extraction}

Study design

Adapted from Figueiras [18], study designs were classified as follows: (1) before/after study; (2) non-randomized controlled study without cross-contamination control; (3) non-randomized controlled study with cross-contamination control; (4) randomized controlled study without crosscontamination control; and (5) randomized controlled study without cross-contamination control. Where authors reported the different groups as being in workplaces that were geographically far apart, the study was deemed to have cross-contamination control; and where no mention was made of distance between groups or specific implementation of cross-contamination control, the study was deemed to be without such control.

\section{Target disease}

In cases where studies identified specific diseases in which interventions were made to improve antibiotic use, this was recorded.

\section{Type of intervention}

Educational interventions include any attempt to persuade physicians to modify their practice performance by communicating clinical information strategies [19] and by communication skills training [13]. Strategies that were purely administrative or applied incentives or coercion were excluded from this definition of educational interventions. In our review, we only included studies that assessed educational interventions. However, in studies in which these types of interventions were associated with others, we extracted data on all strategies. Consequently, interventions were classified into the following categories, adapted from Davis [19] and Figueiras [18]: (1) dissemination of printed/audiovisual educational materials (mailed printed matter; protocols and guidelines; self-instruction materials; drug bulletins); (2) group education, including group-session rounds, conferences, lectures, seminars, and tutorials; (3) feedback of physician prescribing patterns (individually, or including a comparison between these patterns and peer behavior and/or accepted standards), or feedback of patient-specific lists of prescribed medication; (4) individual outreach visits; (5) reminders at the time of prescribing; (6) computer-assisted decisionmaking systems; (7) formulary control/restrictive formulary process; (8) patient education (pamphlets); (9) patient education (videotapes); (10) workshops on rapid tests/ introduction of Rapid Antigen Detection Testing (RADT) in consulting offices; (11) enforcement of regulations; (12) prescription feedback, with recommendations to modify it made by pharmacists and/or infectious-disease physicians; (13) financial incentives.

\section{Baseline and follow-up}

Under this head, we included the period during which outcomes were measured (baseline, intervention period and follow-up). 


\section{Analysis}

Studies were classified into different categories, namely: (1) comparison of post-intervention values between groups; (2) comparison of pre- and post-intervention values within each group; (3) comparison of pre- and post-intervention values between groups; (4) comparison of follow-up values between groups; (5) comparison of pre-, post, and follow-up values within each group; and, (6) comparison of pre-, post- and follow-up values between groups.

\section{Statistical tests}

We collected data yielded by statistical tests used to assess the effectiveness of interventions.

\section{Results}

The results extracted from studies consisted of changes in: total antibiotics prescribed/dispensed (T); choice of appropriate antibiotics/adherence to antibiotic guidance according to guideline algorithms, including dosages and administration routes (Ga); attitudes and behavior (At/ Bh); quality of pharmacy practice (Qph).

Study results were classified as: positive $(+)$, if reported as positive or if changes in outcomes measured were statistically significant; partially positive $( \pm)$, if reported as positive for some variables and negative for others; and negative (-), if reported as negative.

\section{Results and discussion}

\section{Selection of papers}

The search yielded a total of 90,350 Abstracts, 47,535 of which were potentially eligible for inclusion. A reading of the titles and abstracts led to an initial selection of 571 papers for full-text analysis; of these, 65 were then selected, made up of 40 primary- and 25 hospital-care studies. After a search of the references cited, 7 papers were added to the primary-care and 6 to the hospital-care studies. A total of 78 papers were included, 47 primary- [20-66] and 31 hospital-care interventions [3,67-96] (Figure 1)

\section{Interventions in primary care professionals}

In the studies analyzed (Table 1), educational interventions in primary care mainly targeted physicians, and outcomes were assessed by reference to the total antibiotic prescription or appropriate antibiotic prescription rates. Educational interventions in pharmacists occurred in 8 studies $[25,32,33,42,44,50,52,66]$, though in 6 cases the interventions covered both pharmacists and physicians. In 21 studies [20,22,23,25,31$33,35-37,39,43,45,46,50-53,55,57,60]$, the interventions were extended to patients and their caregivers or general population.

In primary care (table 2), 33 studies (70\%) [20-24, $26,27,29,30,32-39,41,44,45,47,48,50,51,53,54,56,58-61,63,-$ 65 ] focused on the use of antibiotics in respiratory infections, and one focused on the use of antibiotics in infectious diseases and other infections (urinary infections, skin and soft tissue infections and septicemia) [27]; the remaining $30 \%$ failed to identify any target disease $[25,28,31,40,42,43,46,49,52,55,57,62,64,66]$. Of the 47 papers, 27 (57\%) studied the efficacy/effectiveness of one or more interventions versus non-intervention, using a control group that received the intervention in four studies, dissemination of guideline information in three $[56,57,65]$ and educational sessions on diagnosis of otitis media in one [58]. In this last study, the educational

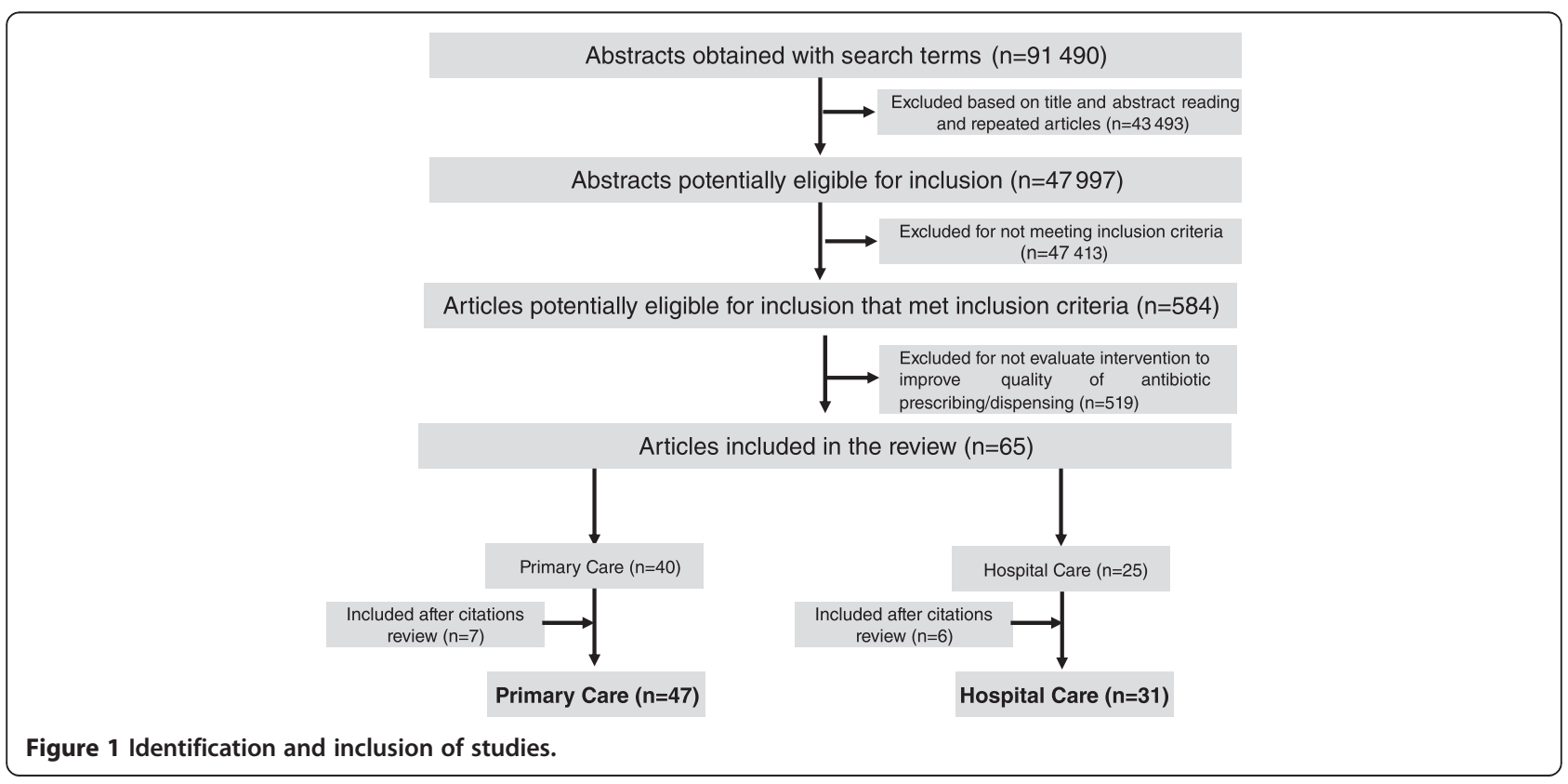


Table 1 Studies analyzing educational interventions in health professionals to improve antibiotic use

\begin{tabular}{|c|c|c|c|c|c|c|}
\hline Author (year) & Country & Allocation unit (a) & Intervention population (b) & Type of patient & Sample size (\%) (b), (c) & Statistical test \\
\hline Dollman, WB (2005) [20] & South Australia & PC & $\mathrm{GPs}, \mathrm{Pa}$ & All & - & Bivariate \\
\hline Hrisos, S (2007) [21] & UK & PC & GPs & - & $340 \mathrm{GPs}$ & Multivariate Bivariate \\
\hline Hennessy, TW (2002) [22] & USA (Alaska) & PC & $\mathrm{Py}, \mathrm{Pa}, \mathrm{O}$ & All & $3144 \mathrm{~Pa}$ & Multivariate Bivariate \\
\hline Rubin, MA (2005) [23] & USA & PC & $\mathrm{Py}, \mathrm{Pa}$ & All & - & Multivariate \\
\hline Naughton, C (2009) [24] & Ireland & PC & GPs & All & 110 GPS & Multivariate \\
\hline Chazan, B (2007) [25] & Israel (Northern) & PC & $\mathrm{Py}, \mathrm{Nu}, \mathrm{Ph}, \mathrm{Pa}$ & All & 200 participants & Bivariate \\
\hline \multirow[t]{2}{*}{ Briel, M (2005) [26] } & Switzerland & PC & Py & Adults & 45 Py & Multivariate Bivariate \\
\hline & & & & & $624 \mathrm{~Pa}$ & \\
\hline Monette, J (2007) [27] & Canada & PC & Py & Geriatric patients & 36 Py & Multivariate \\
\hline Enriquez-Puga, A (2009) [28] & England & PC & $P y, G P s$ & All & 28 practices & Multivariate Bivariate \\
\hline Bjerrum, L (2006) [29] & Spain & PC & GPs & Adults & 17 GPs in IG35 GPs in CG & - \\
\hline \multirow[t]{2}{*}{ Mcisaac, WJ (2002) [30] } & Canada & $\mathrm{PC}$ & GPs & Children Adults & $97 \mathrm{Py}$ & Multivariate Bivariate \\
\hline & & & & & 621 patients & \\
\hline \multirow[t]{2}{*}{ Wheeler, JG (2001) [31] } & USA & PC & $\mathrm{Py}, \mathrm{Pa}$ & Pediatric patients & 16 Py & Bivariate \\
\hline & & & & & 771 parents & \\
\hline \multirow[t]{2}{*}{ Juzych, NS (2005) [32] } & USA & PC & $\mathrm{Py}, \mathrm{Ps}, \mathrm{Nu}, \mathrm{Ph}, \mathrm{Pa}$ & Adults Children & $12 P y+9$ Ps in IG & Univariate \\
\hline & & & & & $6 \mathrm{Py}+9 \mathrm{Ps}$ in CG & \\
\hline \multirow[t]{2}{*}{ Smeets, HM (2009) [33] } & Netherlands & $P C$ & $\mathrm{GPs}, \mathrm{Ph}, \mathrm{Pa}$ & - & 131 practices in IG & Multivariate Bivariate \\
\hline & & & & & 127 practices in CG & \\
\hline Mandryk, JA (2006) [34] & Australia & PC & GPs & - & - & Multivariate \\
\hline Stille, CJ (2008) [35] & USA & PC & $\mathrm{Py}, \mathrm{Pa}$ & Pediatric patients & 168 Py & Multivariate Bivariate \\
\hline \multirow[t]{2}{*}{ Finkelstein, JA (2001) [36] } & USA & PC & $\mathrm{Py}, \mathrm{Pa}$ & $<6$ years & 14468 Pa (pre-) & Multivariate Bivariate \\
\hline & & & & & $13461 \mathrm{~Pa}$ (post-) & \\
\hline \multirow[t]{3}{*}{ Altiner, A (2007) [37] } & Germany & PC & GPs, Pa & $\geq 16$ years & 104 GPs (pre-) & Multivariate \\
\hline & & & & & $28 \mathrm{GPs}+787 \mathrm{~Pa}$ in CG & \\
\hline & & & & & $33 \mathrm{GPs}+920 \mathrm{~Pa}$ in IG & \\
\hline \multirow[t]{2}{*}{ Légaré, F (2010) [38] } & Canada & PC & Py & All & 18 Py in IG + 15 Py in IG & Multivariate \\
\hline & & & & & $245 \mathrm{~Pa}$ in IG + $214 \mathrm{~Pa}$ In CG & \\
\hline Kiang, KM (2005) [39] & USA & PC & $\mathrm{Py}, \mathrm{GPs}, \mathrm{Ps}, \mathrm{Nu}, \mathrm{Pa}, \mathrm{O}$ & Adults and pediatric patients & 1800 Py & Multivariate \\
\hline \multirow[t]{2}{*}{ Mohagheghi, MA (2005) [40] } & Iran & PC & GP & Adults & 40 GPs in $C G$ & - \\
\hline & & & & & 40 GPs in IG & \\
\hline Irurzun, C (2005) [41] & Argentina & PC & Py & $\geq 15$ years & 19 Py & Bivariate \\
\hline Chalker, J (2005)[42] & Vietnam and Thailand & Pharmacy & $\mathrm{Ph}$ & - & 124 pharmacies & Multivariate \\
\hline
\end{tabular}


Table 1 Studies analyzing educational interventions in health professionals to improve antibiotic use (Continued)

\begin{tabular}{|c|c|c|c|c|c|c|}
\hline Finkelstein, JA (2008) [43] & USA & $P C$ & $\mathrm{Py}, \mathrm{Pa}$ & $\leq 6$ years & 223135 person/years & Multivariate \\
\hline Chuc, NTK (2002) [44] & Vietnam & Pharmacy & $\mathrm{Ph}$ & - & 58 pharmacies & Bivariate \\
\hline \multirow[t]{2}{*}{ Belongia, EA (2001) [45] } & USA & PC & $\mathrm{Ps}, \mathrm{Pa}$ & Children & 109 Py in IG & Multivariate Univariate \\
\hline & & & & & 52 in CG & \\
\hline Belongia, EA (2005) [46] & USA & PC & $P y, P s, P a$ & - & $12790 \mathrm{Py}$ & Multivariate Univariate \\
\hline Greene, RA (2004) [47] & USA & PC & Py, Ps & Adults Children & $900 \mathrm{Py}$ and $\mathrm{Pa}$ & Bivariate \\
\hline Teng, CL (2007) [48] & Malaysia & PC & GPs & - & $29 \mathrm{GPs}$ & Bivariate \\
\hline Awad, Al (2006) [49] & Sudan & PC & GPs & - & $1800 \mathrm{~Pa}$ & Bivariate \\
\hline Welschen, I (2004) [50] & Netherlands & PC & GPs, Ph, $\mathrm{Pa}, \mathrm{O}$ & - & 89 GPS & Bivariate \\
\hline \multirow[t]{2}{*}{ Gonzales, R (2004) [51] } & USA & PC & $\mathrm{Py}, \mathrm{Pa}$ & Elderly & 51 office practice in CG & Multivariate \\
\hline & & & & & 4 office practices in IG & \\
\hline $\begin{array}{l}\text { Colomina Rodríguez, J } \\
\text { (2010) [52] }\end{array}$ & Spain & PC & $\mathrm{Py}, \mathrm{Ph}, \mathrm{Pa}, \mathrm{O}$ & All & - & Bivariate \\
\hline \multirow[t]{2}{*}{ Hickman, DE (2003) [53] } & USA & PC & $\mathrm{Py}, \mathrm{Nu}, \mathrm{Pa}$ & Adults & - & Bivariate \\
\hline & & & & Children & & \\
\hline \multirow[t]{2}{*}{ Coenen, S (2004) [54] } & Belgium & PC & GPs & Adults & $42 \mathrm{GPs}$ in IG & Multivariate Bivariate \\
\hline & & & & & 43 GPs in CG & \\
\hline Perz, JF (2002) [55] & USA & PC & $P y, P s, P a$ & Pediatric patients & 464200 person-years & Multivariate \\
\hline Sondergaard, J (2003) [56] & Denmark & PC & Py & - & 299 GPs & Bivariate \\
\hline \multirow[t]{2}{*}{ Doyne, EO (2004) [57] } & USA & PC & $\mathrm{Ps}, \mathrm{Pa}$ & Pediatric patients & 6 practices - IG & Multivariate \\
\hline & & & & & 6 practices - CG & \\
\hline \multirow[t]{2}{*}{ Bauchner, H (2006) [58] } & USA & PC & Ps & Children (3-36 months) & $1368 \mathrm{~Pa}-\mathrm{IG}$ & Multivariate Bivariate \\
\hline & & & & & $1138 \mathrm{~Pa}-\mathrm{CG}$ & \\
\hline \multirow[t]{2}{*}{ Christakis, DA (2001) [59] } & USA & PC & Ps, Nu, O & Children & 16 providers - IG & Bivariate \\
\hline & & & & & 12 providers - CG & \\
\hline Smabrekke, L (2002) [60] & Norway & PC & $\mathrm{Ps}, \mathrm{Nu}, \mathrm{Pa}$ & Children ( $1-5$ years) & $819 \mathrm{~Pa}$ & Bivariate \\
\hline Bjerrum, L (2011) [61] & Several & PC & GP & Adults & 47011 & - \\
\hline Regev-Yochay, G (2011) [62] & Israel & PC & GP & Children & 3636 & Multivariate \\
\hline \multirow[t]{2}{*}{ Llor, C (2011) [63] } & Spain & PC & GP & - & 235 (full) & Univariate Multivariate \\
\hline & & & & & 97 (partial) & \\
\hline Weiss, K (2011) [64] & Canada & PC & $\mathrm{GP}$ & - & All GP & Multivariate \\
\hline Llor, C (2011) [65] & Spain & PC & GP & Adults (14-60 years) & 10 first patients & - \\
\hline McKay, RM (2011) [66] & Canada & $P C$ & $\mathrm{Py}, \mathrm{Ph}, \mathrm{O}$ & - & - & Bivariate \\
\hline Deuster, S (2010) [3] & Switzerland & $\mathrm{HC}$ & Py & Adults & $292 \mathrm{~Pa}$ & Bivariate \\
\hline
\end{tabular}


Table 1 Studies analyzing educational interventions in health professionals to improve antibiotic use (Continued)

\begin{tabular}{|c|c|c|c|c|c|c|}
\hline \multirow[t]{2}{*}{ Chang, MT (2006) [67] } & \multirow[t]{2}{*}{ Taiwan } & \multirow[t]{2}{*}{$\mathrm{HC}$} & \multirow[t]{2}{*}{ GPS } & \multirow[t]{2}{*}{-} & 5046 Pa (pre-) & \multirow[t]{2}{*}{ Bivariate } \\
\hline & & & & & $5054 \mathrm{~Pa}$ (post-) & \\
\hline Naughton, BJ (2001) [68] & USA & $\mathrm{HC}$ & $\mathrm{Py}, \mathrm{Nu}$ & Geriatric patients & 350 episodes & Bivariate \\
\hline Lutters, M (2004) [69] & Switzerland & $\mathrm{HC}$ & Py & Geriatric patients & $3383 \mathrm{~Pa}$ & Bivariate \\
\hline Loeb, M (2005) [70] & Canada and USA & $\mathrm{HC}$ & $\mathrm{Py}, \mathrm{Nu}$ & Geriatric patients & 4217 residents & Bivariate \\
\hline Lesprit, P (2009) [71] & France & $\mathrm{HC}$ & Py & - & $786 \mathrm{~Pa}$ & Bivariate \\
\hline \multirow[t]{2}{*}{ Akter, SFU (2009) [72] } & \multirow[t]{2}{*}{ Bangladesh } & \multirow[t]{2}{*}{$\mathrm{HC}$} & \multirow[t]{2}{*}{ Py } & \multirow[t]{2}{*}{ Pediatric patients } & 2171 Pa (pre-) & \multirow[t]{2}{*}{ Bivariate } \\
\hline & & & & & 1295 Pa (post-) & \\
\hline \multirow[t]{3}{*}{ Paul, M (2006) [73] } & Israel & \multirow[t]{3}{*}{$\mathrm{HC}$} & \multirow[t]{3}{*}{ Py } & \multirow[t]{3}{*}{ Adults } & 1203 Pa (pre-) & \multirow[t]{3}{*}{ Bivariate } \\
\hline & Germany & & & & & \\
\hline & Italy & & & & $\begin{array}{l}2326 \mathrm{~Pa} \text { (post-) (1245 IG and } \\
1801 \text { CG) }\end{array}$ & \\
\hline Camins, BC (2009) [74] & USA & $\mathrm{HC}$ & Py (internists) & - & 784 new prescriptions & Multivariate Bivariate \\
\hline Westphal, JF (2010) [75] & France & $\mathrm{HC}$ & Py & - & $\begin{array}{l}471 \text { cases of pneumonia } 104 \\
\text { (pre-); } 367 \text { (post-) }\end{array}$ & Bivariate \\
\hline \multirow[t]{2}{*}{ Mullet, CJ (2001) [76] } & \multirow[t]{2}{*}{ USA } & \multirow[t]{2}{*}{$\mathrm{HC}$} & \multirow[t]{2}{*}{$\mathrm{PS}, \mathrm{Nu}$} & \multirow[t]{2}{*}{ Pediatric $>6$ months } & 809 Pa (pre-) & \multirow[t]{2}{*}{ Bivariate } \\
\hline & & & & & $949 \mathrm{~Pa}$ (post-) & \\
\hline von Gunten, V (2005) [77] & Switzerland & $\mathrm{HC}$ & Py & - & $1200 \mathrm{~Pa}$ & Multivariate Bivariate \\
\hline Ansari, F (2003) [78] & UK & $\mathrm{HC}$ & Py & - & 40 medical and surgical wards & Multivariate \\
\hline Kisuule, F (2008) [79] & USA & $\mathrm{HC}$ & $\mathrm{Py}, \mathrm{Nu}$ & - & 17 hosp. practitioners & Bivariate \\
\hline \multirow[t]{3}{*}{ Halm, EA (2004) [80] } & \multirow[t]{3}{*}{ USA } & \multirow[t]{3}{*}{$\mathrm{HC}$} & \multirow[t]{3}{*}{$\mathrm{Py}, \mathrm{Nu}, \mathrm{Pa}, \mathrm{O}$} & \multirow[t]{3}{*}{ Adults } & 2094 cases & \multirow[t]{3}{*}{ Bivariate } \\
\hline & & & & & 1013 (pre-) & \\
\hline & & & & & 1081 (post-) & \\
\hline López-Medrano, F (2005) [81] & Spain & $\mathrm{HC}$ & Py & - & 1280 treatments & Bivariate \\
\hline Agwu, AL (2008) [82] & USA & $\mathrm{HC}$ & Py, Ps & Children & - & Bivariate \\
\hline Barenfanger, J (2001) [83] & USA & HC Pharmacy & $\mathrm{Ph}$ & - & $378 \mathrm{~Pa}(188 \mathrm{IG}$ and $190 \mathrm{CG})$ & Bivariate \\
\hline Rüttiman, S (2004) [84] & Switzerland & $\mathrm{HC}$ & Py & Adults & $500 \mathrm{~Pa}$ & Bivariate \\
\hline Martin, C (2005) [85] & USA & $\mathrm{HC}$ & Py & - & - & - \\
\hline Solomon, DH (2001) [86] & USA & $\mathrm{HC}$ & Py & - & $4500 \mathrm{~Pa}$ & Bivariate \\
\hline Fowler, S (2007) [87] & UK & $\mathrm{HC}$ & Py & Elderly $\geq 80$ years & 6129 admissions & Multivariate \\
\hline Sintchenko, V (2005) [88] & Australia & $\mathrm{HC}$ & Py & - & 12 internists & Bivariate \\
\hline Yong, MK (2010) [89] & Australia & $\mathrm{HC}$ & Py & - & - & Bivariate \\
\hline \multirow[t]{2}{*}{ Meyer, E (2010) [90] } & Germany & $\mathrm{HC}$ & Py & Adults & 4684 Pa (pre-) & Multivariate \\
\hline & & & & & $7203 \mathrm{~Pa}$ (post-) & \\
\hline
\end{tabular}


Table 1 Studies analyzing educational interventions in health professionals to improve antibiotic use (Continued)

Thursky, KA (2006) [91] Australia

$\mathrm{HC}$

Petterson, E (2011) [92]

Tangden, T (2011) [93]

Sweeden

Sweeden

$\mathrm{HC}$

$\mathrm{HC}$

Py

$\mathrm{Nu}, \mathrm{Py}$

Py

$489 \mathrm{~Pa}$ (pre-)

$497 \mathrm{~Pa}$ (post-)

Talpaert, MJ (2011) [94]

Bevilacqua, S (2011) [95]

UK

Shen, J (2011) [96]

France

China

$\mathrm{HC}$

$\mathrm{HC}$

$\mathrm{HC}$

Py

Py

Py

(a) $\mathrm{PC}$ - primary care; $\mathrm{HC}$ - hospital care.

(b) GPs - general practitioners; Ps - pediatrics; Py - physicians; Pa - patients or their caregivers; Ph - pharmacists; $\mathrm{Nu}$ - nurses; O - others.

(c) CG - control group; IG - intervention group. 
Table 2 Interventions to improve antibiotic use in primary care

\begin{tabular}{|c|c|c|c|c|c|c|c|c|}
\hline \multirow[t]{2}{*}{ Author (year) } & \multirow{2}{*}{$\begin{array}{l}\text { Study } \\
\text { design (a) }\end{array}$} & \multicolumn{2}{|l|}{ Program description } & \multicolumn{3}{|c|}{ Baseline and follow-up } & \multirow{2}{*}{$\begin{array}{l}\text { Analysis } \\
\text { (e) }\end{array}$} & \multirow[t]{2}{*}{ Results (f) } \\
\hline & & Disease (b) & $\begin{array}{l}\text { Intervention type } \\
(c, d)\end{array}$ & Baseline & Intervention period & Follow-up & & \\
\hline \multirow[t]{2}{*}{ Dollman, WB (2005) [20] } & \multirow[t]{2}{*}{1} & \multirow[t]{2}{*}{ URTI } & IG: 1, 2, 8 & \multirow[t]{2}{*}{5 months } & \multirow[t]{2}{*}{5 months } & \multirow[t]{2}{*}{-} & \multirow[t]{2}{*}{2} & \multirow[t]{2}{*}{$\mathrm{T}(+)$} \\
\hline & & & CG: 0 & & & & & \\
\hline \multirow[t]{4}{*}{ Hrisos, S (2008) [21] } & \multirow[t]{4}{*}{4} & \multirow[t]{4}{*}{ URTI } & IG1: 3 & \multirow[t]{4}{*}{-} & \multirow[t]{4}{*}{3 months } & \multirow[t]{4}{*}{-} & \multirow[t]{4}{*}{3} & \multirow[t]{4}{*}{ At/Bh (+) } \\
\hline & & & IG2: 3 & & & & & \\
\hline & & & $|G|+2: 3$ & & & & & \\
\hline & & & CG: 0 & & & & & \\
\hline \multirow[t]{2}{*}{ Hennessy, TW (2002) [22] } & \multirow[t]{2}{*}{3} & \multirow[t]{2}{*}{ RTI } & IG: 8,2 & \multirow[t]{2}{*}{2 months } & \multirow{2}{*}{$\begin{array}{l}12 \text { months ( } 6 \text { each } \\
\text { year of intervention) }\end{array}$} & \multirow[t]{2}{*}{2 months } & \multirow[t]{2}{*}{2,3} & $\mathrm{~T}(+)$ \\
\hline & & & CG: 0 & & & & & \\
\hline Rubin, MA (2005) [23] & 2 & URTI & IG: $1,2,8,9$ & 6 months & 6 months & - & 2,3 & $\mathrm{~T}(+)$ \\
\hline & & & CG: 0 & & & & & $\mathrm{Ga}(+)$ \\
\hline Naughton, C (2009) [24] & 4 & RTI & IG1: 3,4 & 12 months & - & 12 months & 2,3 & $\mathrm{~T}(+)(-)^{\mathrm{a}}$ \\
\hline & & & IG2: 3 & & & & & $\mathrm{Ga}(+)(-)^{\mathrm{a}}$ \\
\hline Chazan, B (2007) [25] & 1 & Infectious disease & IG1: 1,2 & 4 months & 4 months & - & 2,3 & $\mathrm{~T}(+)$ \\
\hline & & & IG2: 1, 2, 8 & & & & & \\
\hline Briel, M (2005) [26] & 4 & ARTI & IG1: 1,2 & - & 5 months & - & 1 & $\mathrm{~T}(+)$ \\
\hline & & & IG2: 1,2 & & & & & \\
\hline & & & CG: 0 & & & & & \\
\hline Monette, J (2007) [27] & 4 & Lower RTI & IG: 1,3 & 3 months & $2 \times 3$ months & 3 months & 5,6 & $\mathrm{Ga}(+)$ \\
\hline & & UTI & CG: 0 & & & & & \\
\hline & & $\begin{array}{l}\text { Skin and soft-tissue } \\
\text { infections septicemia }\end{array}$ & & & & & & \\
\hline Enriquez-Puga, A (2009) [28] & 4 & - & IG: $1,3,4$ & $\begin{array}{l}2 \text { periods of } \\
6 \text { months }\end{array}$ & 6 months & 24 months & 5,6 & $\mathrm{Ga}(-)$ \\
\hline Bjerrum, L (2006) [29] & 2 & RTI & IG: $2,3,10$ & 3 weeks during & 3 weeks during 3 months & - & 1,2 & $T(+)$ \\
\hline & & & CG: 0 & 3 months & & & & $\mathrm{Ga}(+)$ \\
\hline Mcisaac, WJ (2002) [30] & 4 & Sore throat & IG: 1,5 & - & - & - & 1 & $\mathrm{~T}(-)$ \\
\hline & & & CG: 0 & & & & & $\mathrm{Ga}(-)$ \\
\hline Wheeler, JG (2001) [31] & 1 & Viral infections & IG: $2,8,9$ & 1 week & 3 weeks during 3 years & 6 months (qualitative) & 3 & $\mathrm{~T}(-)$ \\
\hline & & & & & & & & $\mathrm{At} / \mathrm{Bh}(+)$ \\
\hline Juzych, NS (2005) [32] & 3 & URTI & IG: 1, 2, 8 & 4.5 months & 4.5 months & - & 2,3 & $\mathrm{~Pa}(+)(-)^{\mathrm{b}}$ \\
\hline & & & CG: 0 & & & & & $\mathrm{~T}(+)$ \\
\hline Smeets, HM (2009) [33] & 2 & RTI & IG: $2,3,8$ & 6 months & 6 months & & 5,6 & $\mathrm{~T}(-)$ \\
\hline
\end{tabular}


Table 2 Interventions to improve antibiotic use in primary care (Continued)

\begin{tabular}{|c|c|c|c|c|c|c|c|c|}
\hline & & & CG: 0 & & & $\begin{array}{l}6 \text { months } \\
\text { (one year later) }\end{array}$ & & $\mathrm{Ga}(-)$ \\
\hline \multirow[t]{2}{*}{ Mandryk, JA (2006) [34] } & \multirow[t]{2}{*}{1} & \multirow[t]{2}{*}{ URTI } & \multirow[t]{2}{*}{ IG: $1,2,3,4$} & \multirow[t]{2}{*}{33 months } & \multirow[t]{2}{*}{51 months } & \multirow[t]{2}{*}{ - } & \multirow[t]{2}{*}{2} & $\mathrm{Ga}(+)$ \\
\hline & & & & & & & & $\mathrm{T}(+)$ \\
\hline \multirow[t]{2}{*}{ Stille, CJ (2008) [35] } & \multirow[t]{2}{*}{4} & \multirow[t]{2}{*}{ RTI } & IG: $1,2,8$ & \multirow[t]{2}{*}{-} & \multirow[t]{2}{*}{-} & \multirow[t]{2}{*}{6 months } & \multirow[t]{2}{*}{1} & \multirow[t]{2}{*}{$\mathrm{At} / \mathrm{Bh}(+)(-)^{\mathrm{C}}$} \\
\hline & & & CG: 0 & & & & & \\
\hline \multirow[t]{5}{*}{ Finkelstein, JA (2001) [36] } & \multirow[t]{5}{*}{4} & Otitis media & IG: $1,2,3,8$ & \multirow[t]{5}{*}{12 months } & \multirow[t]{5}{*}{12 months } & \multirow[t]{5}{*}{ - } & \multirow[t]{5}{*}{2,3} & \multirow[t]{5}{*}{$\mathrm{T}(+)$} \\
\hline & & Pharyngitis & CG: 0 & & & & & \\
\hline & & Sinusitis & & & & & & \\
\hline & & Cold & & & & & & \\
\hline & & Bronchitis & & & & & & \\
\hline \multirow[t]{2}{*}{ Altiner, A (2007) [37] } & \multirow[t]{2}{*}{4} & \multirow[t]{2}{*}{ Acute cough } & IG: 4,8 & \multirow[t]{2}{*}{3 months } & \multirow[t]{2}{*}{-} & \multirow{2}{*}{$\begin{array}{l}3 \text { months after } \\
6 \text { weeks } 3 \text { months } \\
\text { after } 1 \text { year after }\end{array}$} & 5,6 & $\mathrm{~T}(+)$ \\
\hline & & & CG: 0 & & & & & \\
\hline Légaré, F (2010) [38] & 4 & Acute Rl & IG: 1,2 & - & - & - & 2,3 & $\mathrm{~T}(+)$ \\
\hline & & & CG: 0 & & & & & \\
\hline Kiang, KM (2005) [39] & 1 & Respiratory & IG: $1,2,8$ & - & - & - & 2,3 & $\mathrm{Ga}(+)$ \\
\hline & & Illnesses & & & & & & $\mathrm{At} / \mathrm{Bh}(+)$ \\
\hline Mohagheghi, MA [40] & 4 & - & IG: 2 & 60 months & - & 3 months afterwards & 2,3 & $\mathrm{~T}(+)(-)^{\mathrm{d}}$ \\
\hline & & & CG: 0 & & & 1 year atterwaras & & \\
\hline Irurzun, C (2005) [41] & 1 & Pharyngitis and & IG: $1,2,3,4,10$ & - & 12 months & - & 2 & $\mathrm{~T}(+)$ \\
\hline & & & & & & & & $\mathrm{Ga}(+)$ \\
\hline Chalker, J (2005) [42] & 5 & - & IG: 2, 4, 11 & - & - & $3 \times 3$ months (one & 1 & $\mathrm{~T}(+)(-)^{\mathrm{e}}$ \\
\hline & & & CG: 0 & & & intervention) & & \\
\hline Finkelstein, JA (2008) [43] & 4 & - & IG: $1,2,3,8$ & 24 months & 6 months during & - & 2,3 & $T(+)(-)^{f}$ \\
\hline & & & CG: 0 & & & & & $\mathrm{Ga}(+)$ \\
\hline Chuc, NTK (2002) [44] & 4 & ARTI & IG: 2, 4, 11 & - & - & - & 2,3 & $\mathrm{~T}(+)$ \\
\hline & & & & & & & & Qh $(+)$ \\
\hline Belongia, EA (2001) [45] & 3 & ARTI & IG: $1,2,8$ & 6 months & - & $\begin{array}{l}6 \text { months (every } \\
\text { two years) }\end{array}$ & 7,8 & $\mathrm{~T}(+)$ \\
\hline Belongia, EA (2005) [46] & 2 & - & IG: $1,2,8,9$ & 12 months & 48 months & - & 3,4 & $\mathrm{~T}(+)(-)^{\mathrm{g}}$ \\
\hline Greene, RA (2004) [47] & 1 & Acute sinusitis & IG: $1,2,3,13$ & 22 months & 14 months & - & 2 & $\mathrm{Ga}(+)$ \\
\hline & & & & & & & & $\mathrm{T}(+)$ \\
\hline Teng, CL (2007) [48] & 2 & URTI and others & IG: 1, 2, 4 & 3 months & _ & 3 months & 2 & $\mathrm{~T}(+)$ \\
\hline
\end{tabular}


Table 2 Interventions to improve antibiotic use in primary care (Continued)

\begin{tabular}{|c|c|c|c|c|c|c|c|c|}
\hline \multirow[t]{4}{*}{ Awad, Al (2006) [49] } & \multirow[t]{4}{*}{4} & \multirow[t]{4}{*}{-} & CG: 0 & \multirow[t]{4}{*}{-} & \multirow[t]{4}{*}{-} & \multirow{4}{*}{$\begin{array}{l}1 \text { and } 3 \text { months } \\
\text { afterwards }\end{array}$} & \multirow[t]{4}{*}{2,3} & $\mathrm{~T}(+)^{\mathrm{h}}$ \\
\hline & & & $|G|: 1,3$ & & & & & \multirow[t]{3}{*}{$\mathrm{Ga}(+)$} \\
\hline & & & IG2: 2, 3 & & & & & \\
\hline & & & IG3: 3,4 & & & & & \\
\hline Welschen, I (2004) [50] & 4 & ARTI & IG: $1,2,3,8$ & 3 months & - & 3 months & 2,3 & $T(+)$ \\
\hline Gonzales, R (2004) [51] & 2 & ARTI & $\mathrm{IG}: 1,8$ & 4 months & 4 months (study period) & & 2,3 & $\mathrm{~T}(+)(-)^{\mathrm{i}}$ \\
\hline \multirow[t]{2}{*}{ Colomina Rodríguez, J (2010) [52] } & \multirow[t]{2}{*}{1} & \multirow[t]{2}{*}{ - } & IG: $1,2,6,8$ & \multirow[t]{2}{*}{48 months } & \multirow[t]{2}{*}{36 months } & \multirow[t]{2}{*}{24 months } & \multirow[t]{2}{*}{5} & $T(+)$ \\
\hline & & & & & & & & $\mathrm{Ga}(+)$ \\
\hline \multirow[t]{2}{*}{ Hickman, DE (2003) [53] } & \multirow[t]{2}{*}{4} & \multirow[t]{2}{*}{ Acute bronchitis } & IG: $1,2,8$ & \multirow[t]{2}{*}{6 months } & \multirow[t]{2}{*}{-} & \multirow[t]{2}{*}{6 months } & \multirow[t]{2}{*}{2,3} & \multirow[t]{2}{*}{$T(+)$} \\
\hline & & & CG: 0 & & & & & \\
\hline \multirow[t]{2}{*}{ Coenen, S (2004) [54] } & \multirow[t]{2}{*}{4} & \multirow[t]{2}{*}{ Acute cough } & IG: 1,4 & \multirow[t]{2}{*}{3 months } & \multirow{2}{*}{$\begin{array}{l}1 \text { month (without } \\
\text { outcomes) }\end{array}$} & - & 2,3 & $\mathrm{~T}(+)$ \\
\hline & & & IG: 0 & & & & & $\mathrm{Ga}(+)(-)^{\mathrm{j}}$ \\
\hline Perz, JF (2002) [55] & 1 & - & IG: $1,2,8,9$ & 12 months & 12 months & 12 months & 5,6 & $\mathrm{~T}(+)$ \\
\hline & & & CG: 0 & & & & & \\
\hline Sondergaard, J (2003) [56] & 4 & $\mathrm{RTI}$ & IG: 1,3 & 3 periods of & 3 periods of 3 months & 3 months & 2,3 & $\mathrm{~T}(-)$ \\
\hline & & & CG: 1 & 3 months & & (not shown) & & $\mathrm{Ga}(-)$ \\
\hline Doyne, EO (2004) [57] & 4 & - & IG: $1,2,3,8$ & 12 months & 12 months & - & 2,3 & $\mathrm{~T}(+)(-)^{\mathrm{k}}$ \\
\hline & & & CG1: 1, 3 & & & & & \\
\hline & & & CG: 1 & & & & & \\
\hline Bauchner, H (2006) [58] & 5 & Acute otitis & IG: 1, 2, 3 & - & - & - & 1 & $\mathrm{Ga}(+)(-)^{\mathrm{L}}$ \\
\hline & & & CG: 2 & & & & & \\
\hline Christakis, DA (2001) [59] & 4 & Acute otitis & IG: 6 & 7 months & 8 months & - & 2,3 & $\mathrm{~T}(-)$ \\
\hline & & media & CG: 0 & & & & & $\mathrm{Ga}(+)$ \\
\hline Småbrekke, L (2002) [60] & 2 & Acute otitis & IG: $1,2,8$ & 4 months & 4 months & - & 2,3 & $\mathrm{~T}(+)$ \\
\hline & & & CG: 0 & & & & & $\mathrm{Ga}(+)$ \\
\hline Bjerrum, L (2011) [61] & 1 & $\mathrm{RTI}$ & $\mathrm{IG}=2,3,9,10$ & 3 weeks & 3 weeks ( $x 1$ year) & - & 2,3 & $\mathrm{~T}(+)$ \\
\hline & & & & (x2years) & & & & $\mathrm{Ga}(+)$ \\
\hline Regev-Yochay, G (2011) [62] & 4 & - & $I G=2$ & 2 years & 1 year & - & 2,3 & $\mathrm{~T}(+)$ \\
\hline & & & $C G=0$ & & & & & $\mathrm{Ga}(+)$ \\
\hline Llor, C (2011) [63] & 4 & Pharyngitis & $|\mathrm{G}|=2,8,10$ & 15 days & 15 days & - & 2,3 & $T(+)$ \\
\hline & & & $\begin{array}{l}\mathrm{IG} 2=2,8,10 \\
(\mathrm{sem})\end{array}$ & & & & & \\
\hline Weiss, K (2011) [64] & 1 & - & $\mid \mathrm{G}=1$ & 2 years & 7 years & - & 2,3 & $\mathrm{~T}(+)$ \\
\hline & & & $C G=0$ & & & & & \\
\hline
\end{tabular}


Table 2 Interventions to improve antibiotic use in primary care (Continued)

\begin{tabular}{|c|c|c|c|c|c|c|c|c|}
\hline \multirow[t]{2}{*}{ Llor, C (2011) [65] } & \multirow[t]{2}{*}{4} & \multirow{2}{*}{$\begin{array}{l}\text { Acute } \\
\text { pharyngitis }\end{array}$} & $\mathrm{IG}=1,10$ & \multirow[t]{2}{*}{ - } & \multirow[t]{2}{*}{-} & \multirow[t]{2}{*}{ - } & \multirow[t]{2}{*}{1} & \multirow[t]{2}{*}{$\mathrm{Ga}(+)$} \\
\hline & & & $C G=1$ & & & & & \\
\hline McKay, RM (2011) [66] & 1 & - & $\mathrm{IG}=1,2,8,9$ & 9 years & 3 years & - & 2 & $\mathrm{~Pa}(+)(-)^{\mathrm{m}}$ \\
\hline
\end{tabular}

an[24], significantly positive in post-intervention period but no significant change post-follow-up.

$b$ In [32], while prescriptions for pharyngitis, otitis media and URTI decreased significantly post-intervention, the decrease in the case of bronchitis was not as significant.

II [32], while prescriptions for pharyngitis, otitis media and URTI decreased significantly post-intervention, the decrease in the case of bronchitis was not as significant.
In [35], comparison between attitudes, knowledge and behavior of physicians in the intervention versus the control group showed no significant differences. Physicians in the intervention group reported that they had In [35], comparison between attitudes, knowledge
changed their prescribing in the preceding 3 years.

changed their prescribing in the preceding 3 years.
$d_{I n}[40]$, after one year, there was a reduction in the percentage of antibiotic prescribing in the intervention group but this was not statistically different from the control group.

eIn [42], interventions resulted in improved antibiotic use, which was statistically significant in the Hanoi but not in the Bangkok study.

II [43], there was no significant decrease in one age group (3-24 months).

9 In [56], the reduction in antibiotic prescribing by pediatricians was greater in the control than in the intervention group.

${ }^{h}$ In [49], audit and feedback combined with academic detailing or seminars appeared to be more effective in changing antibiotic prescribing practices than audit and feedback alone.

iIn [51], there was a moderate decrease in total antibiotics prescribed but this was not statistically significant.

IIn [54], appropriate antibiotic prescribing improved post-intervention but did not prove statistically significant.

${ }^{k}$ In [57], the prescribing rate decreased in all groups but there were no statistically significant differences between groups.

In [58], adherence was high though not statistically significant in the intervention group, but, in second episodes there were no differences in adherence, between groups.

$\mathrm{m} / \mathrm{n}$ [66], utilization rates for acute bronchitis are at the same level as when intervention began, but other acute respiratory tract infections declined.

(a) Disease: URTI - upper respiratory tract infections; RTI - respiratory tract infections; ARTI - acute respiratory tract infections; UTI - urinary tract infections.

(b) Study design (SD): (1) before/after studies; (2) - nonrandomized controlled trial without cross-contamination control; (3) - nonrandomized controlled trial with cross-contamination control; (4) - randomized controlled trial without cross-contamination control; (5) - randomized controlled trial with cross-contamination control.

(c) IG - intervention group; CG - control group.

(d) Type of intervention (TI): (0) no intervention; (1) dissemination of printed/audiovisual educational materials (mailed printed matter; protocols and guidelines; self-instruction materials; drug bulletins); (2) group education, including group-session rounds, conferences, lectures, seminars and tutorials; (3) feedback of physician prescribing patterns (individually or including a comparison of these patterns with peer behavior and/ or accepted standards) or feedback of patient-specific lists of prescribed medication; (4) individual outreach visits; (5) reminders at the time of prescribing; (6) computer-assisted decision-making systems; (7) formularyor accepted standards) or feedback of patient-specific lists of prescribed medication; (4) individual outreach visits; (5) reminders at the time of prescribing; (6) computer-assisted decision-making systems; (7) formulary-
control/restrictive formulary process; (8) patient education (pamphlets); (9) patient education (videotapes); (10) workshops on rapid tests / introduction of Rapid Antigen Detection Tests (RADTs) in consulting offices; (11) enforcement of regulations; (12) prescription feedback with recommendations to modify it by pharmacists and/or infectious-disease physicians; (13) financial incentives.

(11) enforcement of regulations; (12) prescription feedback with recommendations to modify it by pharmacists and/or infectious-disease physicians; (13) financial incentives.
(e) Type of data-analysis (T): (1) comparison of post-test values between groups; (2) comparison of pre- and post-values within each group; (3) comparison of pre- and post-values between groups; (4) comparison of (e) Type of data-analysis (T): (1) comparison of post-test values between groups; (2) comparison of pre- and post-values within each group; (3) comparison of pre- and post-val
follow-up values between groups; (5) comparison of pre-, post- and follow-up values within each group; (6) comparison of pre-, post- and follow-up values between groups.

follow-up values between groups; (5) comparison of pre-, post- and follow-up values within each group; (6) comparison of pre-, post- and follow-up values between groups. administration; ( $\mathrm{Pa}$ ) prescription rate per disease; (At/Bh) attitudes and behavior; (Qph) quality of pharmacy practice. 
Table 3 Interventions to improve antibiotic use in hospital settings

\begin{tabular}{|c|c|c|c|c|c|c|c|c|}
\hline \multirow[t]{2}{*}{ Author (year) } & \multirow{2}{*}{$\begin{array}{l}\text { Study } \\
\text { design } \\
\text { (a) }\end{array}$} & \multicolumn{2}{|l|}{ Program description } & \multicolumn{3}{|l|}{ Baseline and follow-up } & \multirow[t]{2}{*}{ Analysis (d) } & \multirow[t]{2}{*}{ Results (e) } \\
\hline & & Disease & Intervention type $(b, c)$ & Baseline & Intervention period & Follow-up & & \\
\hline Deuster, S (2010) [3] & 1 & Most common hospital infections & IG: 1,2 & 8 weeks & 8 weeks & 8 weeks (1 year after) & 5 & $\mathrm{Ga}(+)(-)^{\mathrm{a}}$ \\
\hline \multirow[t]{2}{*}{ Chang, MT (2006) [67] } & \multirow[t]{2}{*}{1} & \multirow[t]{2}{*}{-} & \multirow[t]{2}{*}{ IG: 1,7} & \multirow[t]{2}{*}{3 months } & \multirow[t]{2}{*}{3 months } & \multirow[t]{2}{*}{-} & \multirow[t]{2}{*}{2} & $\mathrm{~T}(+)$ \\
\hline & & & & & & & & $\mathrm{Ga}(+)$ \\
\hline \multirow[t]{2}{*}{ Naughton, BJ (2001) [68] } & \multirow[t]{2}{*}{4} & \multirow[t]{2}{*}{ Pneumonia } & IG: 1,2 & \multirow[t]{2}{*}{6 months } & \multirow[t]{2}{*}{6 months } & \multirow[t]{2}{*}{-} & \multirow[t]{2}{*}{2,3} & \multirow[t]{2}{*}{$\mathrm{T}(-)$} \\
\hline & & & CG: 1,2 & & & & & \\
\hline \multirow[t]{2}{*}{ Lutters, M (2004) [69] } & \multirow[t]{2}{*}{1} & \multirow[t]{2}{*}{ RTI and UTI } & IG: 1, 2, 4 & \multirow[t]{2}{*}{12 months } & \multirow[t]{2}{*}{24 months } & - & 2 & $\mathrm{~T}(+)$ \\
\hline & & & & & & & & $\mathrm{Ga}(+)$ \\
\hline Loeb, M (2005) [70] & 4 & UTI & IG: $1,2,4$ & - & - & - & 1 & $\mathrm{~T}(+)$ \\
\hline & & & CG: 0 & & & & & \\
\hline Lesprit, P (2009) [71] & 2 & Various & IG: $1,2,12$ & - & 8 weeks & - & 1 & $\mathrm{Ga}(+)$ \\
\hline & & & CG: 1,2 & & & & & \\
\hline Akter, SFU (2009) [72] & 2 & Common pediatric infections & IG: 2 & 4 months & 4 months & - & 2,3 & $\mathrm{~T}(+)$ \\
\hline & & & & & & & & $\mathrm{Ga}(+)$ \\
\hline Paul, M (2006) [73] & 5 & - & IG: 6 & 7 months & 7 months & - & 1,2 & $\mathrm{Ga}(+)$ \\
\hline & & & CG: 0 & & & & & \\
\hline Camins, BC (2009) [74] & 4 & - & IG: $1,3,4$ & - & 10 months & - & 1 & $\mathrm{Ga}(+)$ \\
\hline & & & CG: 1 (guidelines) & & & & & \\
\hline Westphal, JF (2010) [75] & 1 & Pneumonia & IG: $2,5,6$ & 18 months & 54 months & - & 2 & $G a(+)(-)^{b}$ \\
\hline Mullet, CJ (2001) [76] & 1 & - & IG: 6 & 6 months & 6 months & - & 2 & $\mathrm{~T}(+)(-)^{c}$ \\
\hline & & & & & & & & $\mathrm{Ga}(+)$ \\
\hline von Gunten, V (2005) [77] & 5 & - & $I_{B}: 1$ & 6 months & 6 months & - & 2,3 & $\mathrm{~T}(+)$ \\
\hline & & & $I_{c}: 1,2,12$ & & & & & $\mathrm{Ga}(+)$ \\
\hline & & & $C G_{A}: 0$ & & & & & \\
\hline Ansari, F (2003) [78] & 1 & - & IG: 12 & 24 months & 24 months & - & 2 & $G a(+)(-)^{d}$ \\
\hline & & & & & & & & $\mathrm{T}(+)$ \\
\hline Kisuule, F (2008) [79] & 1 & - & IG: $1,3,4$ & Period until 20 prescriptions & 2 months & 1 month & 2 & $\mathrm{Ga}(+)$ \\
\hline Halm, EA (2004) [80] & 1 & Pneumonia & IG: $1,2,8,9$ & 5 months & - & 5 months & 2 & $\mathrm{Ga}(+)$ \\
\hline López-Medrano, F (2005) [81] & 1 & - & IG: 12 & 12 months & 12 months & - & 2 & $\mathrm{~T}(+)$ \\
\hline & & & & & & & & $\mathrm{Ga}(+)$ \\
\hline Agwu, AL (2008) [82] & 1 & - & IG: 6,12 & 12 months & 12 months & - & 2 & $\mathrm{Ga}(+)$ \\
\hline Barenfanger, J (2001) [83] & 4 & - & IG: 6 & - & 5 months & - & 1 & $\mathrm{~T}(+)$ \\
\hline & & & CG: 0 & & & & & \\
\hline Rüttiman, S (2004) [84] & 1 & - & IG: $1,2,3$ & - & - & - & 2 & $\mathrm{~T}(+) \mathrm{Ga}(+)$ \\
\hline
\end{tabular}


Table 3 Interventions to improve antibiotic use in hospital settings (Continued)

\begin{tabular}{|c|c|c|c|c|c|c|c|c|}
\hline \multirow[t]{3}{*}{ Martin, C (2005) [85] } & 1 & Pneumonia & IG: 1,2 & - & 60 months & - & 2 & $\mathrm{Ga}(+)$ \\
\hline & & Meningitis & & & & & & \\
\hline & & UTI & & & & & & \\
\hline \multirow[t]{2}{*}{ Solomon, DH (2001) [86] } & 4 & - & IG: $1,3,4,12$ & 4 weeks & 18 weeks & - & 2,3 & $\mathrm{Ga}(+)$ \\
\hline & & & CG: 0 & & & & & \\
\hline Fowler, S (2007) [87] & 1 & - & IG: 1,3 & 21 months & 21 months & - & 2 & $\mathrm{Ga}(+)$ \\
\hline Sintchenko, V (2005) [88] & 1 & Intensive care & IG: 6 & 6 months & 6 months & - & 2 & $\mathrm{~T}(+) \mathrm{Ga}(+)$ \\
\hline Yong, MK (2010) [89] & 1 & Intensive care & IG: 6 & 30 months & 54 months & - & 2 & $\mathrm{Ga}(+)$ \\
\hline Meyer, E (2010) [90] & 1 & Intensive care & IG: 2 & 24 months & 36 months & - & 2 & $\mathrm{~T}(+)$ \\
\hline Thursky, KA (2006) [91] & 1 & Intensive care & IG: 2,6 & 6 months & 6 months & - & 2 & $\mathrm{~T}(+) \mathrm{Ga}(+)$ \\
\hline \multirow[t]{2}{*}{ Petterson, E (2011) [92] } & 4 & UTI & $\mathrm{IG}=1,2,3$ & 3 months & 3 months & & 2,3 & $\mathrm{~T}(+)$ \\
\hline & & & $C G=0$ & & & & & $\mathrm{Ga}(+)$ \\
\hline \multirow[t]{2}{*}{ Tangden, T (2011) [93] } & 1 & Pneumonia (Intravenous) & $\mathrm{IG}=1,2$ & 7 years & & 2.5 years & 3 & $\mathrm{~T}(+)$ \\
\hline & & & & & & & & $\mathrm{Ga}(+)(-)^{\mathrm{e}}$ \\
\hline Talpaert, MJ (2011) [94] & 1 & - & $\mathrm{IG}=2$ & 12 months & 12 months & & 3 & $\mathrm{~T}(+)(-)^{f}$ \\
\hline \multirow[t]{2}{*}{ Bevilacqua, S (2011) [95] } & 2 & - & $\mathrm{IG}=3,7,12$ & 12 months & 12 months & & 2,3 & $\mathrm{Ga}(+)$ \\
\hline & & & $C G=0$ & & & & & \\
\hline \multirow[t]{3}{*}{ Shen, J (2011) [96] } & 2 & Bronchitis & $\mathrm{IG}=12$ & & 10 months & & 1 & $\mathrm{Ga}(+)$ \\
\hline & & Community acquired pneumonia & $C G=0$ & & & & & \\
\hline & & Acute exacerbation of COPD & & & & & & \\
\hline
\end{tabular}

an [3], the follow-up analysis showed sustained adherence to guidelines in hospital-acquired pneumonia but a decrease in guideline adherence in the case of UTI.

IIn [75], there was a significant decrease in the proportion of antibiotic orders containing at least one criterion that was not in line with the guideline, but the choice of antibiotics according to the context of acquisition of pneumonia, improvement was not statistically significant.

cIn [76], total of antibiotics used was similar but the number of orders placed per antibiotic course decreased post-intervention.

${ }^{d}$ In [78], there was a significant decrease in use of total and alert antibiotics, except in the case of ceftriaxone and mercapen.

eIn [93], there was a reduction of cefalosporines consumption, but pipiracillin/tazobactan and penicillin increased

In [94], there was a reduction in fluorquinolone and cefalosporine but no significant change total of antibiotics neither clindamicine, amoxiciline and co-amoxclav use.

(a) Disease: URTI - upper respiratory tract infections; RTI - respiratory tract infections; ARTI - acute respiratory tract infections; UTI - urinary tract infections; COPD-Chronic obstructive pulmonary disease.

(b) Study design (SD): (1) before/after studies; (2) - nonrandomized controlled trial without cross-contamination control; (3) - nonrandomized controlled trial with cross-contamination control; (4) - randomized controlled trial without cross-contamination control; (5) - randomized controlled trial with cross-contamination control.

(c) IG - intervention group; CG - control group.

(d) Type of intervention (TI): (0) no intervention; (1) dissemination of printed/audiovisual educational materials (mailed printed matter; protocols and guidelines; self-instruction materials; drug bulletins); (2) group education, including group-session rounds, conferences, lectures, seminars and tutorials; (3) feedback of physician prescribing patterns (individually or including a comparison of these patterns with peer behavior and/ or accepted standards) or feedback of patient-specific lists of prescribed medication; (4) individual outreach visits; (5) reminders at the time of prescribing; (6) computer-assisted decision-making systems; (7) formularycontrol/restrictive formulary process; (8) patient education (pamphlets); (9) patient education (videotapes); (10) workshops on rapid tests / introduction of Rapid Antigen Detection Tests (RADTs) in consulting offices; (11) enforcement of regulations; (12) prescription feedback with recommendations to modify it by pharmacists and/or infectious-disease physicians; (13) financial incentives.

(e) Type of data-analysis (T): (1) comparison of post-test values between groups; (2) comparison of pre- and post-values within each group; (3) comparison of pre- and post-values between groups; (4) comparison of follow-up values between groups; (5) comparison of pre-, post- and follow-up values within each group; (6) comparison of pre-, post- and follow-up values between groups.

(f) Results analyzed (R): (T) total antibiotics prescribed/dispensed; (Ga) choice of appropriate antibiotics/adherence to antibiotic guidance according to guideline algorithms, including dosages and routes of administration; $(\mathrm{Pa})$ prescription rate per pathology: (At/Bh) attitudes and behavior; (Qph) quality of pharmacy practice. 
session in the intervention group included diagnosis of otitis media and information on recommendations for antibiotic use.

Only two studies [51,64], evaluated the efficacy of passive interventions in physicians and in one of them interventions was in combination with educational campaigns directed at patients and their caregivers [51]. All the other studies included active interventions in health professionals (whether or not associated with passive interventions). Three studies $[22,46,66]$, involved active interventions in patients and health professionals, and in four studies $[26,33,50,62]$ the interventions included improvement of doctor-patient communication skills.

Twenty-nine studies (62\%) [20-23,25-27,29,34,36-39, $41,44,45,47-50,52,53,55,60-65]$ reported positive results for all outcomes measured; fourteen studies (30\%) $[24,31,32,35,40,42,43,46,51,54,57-59,66]$ reported positive results for some outcomes, and results that were not statistically influenced by the intervention for others; only four studies [28,30,33,56] failed to report significant post-intervention improvements for all outcomes.

While some studies conducted no post-intervention follow-up of participants [20,21,23,25,26,29,30,32,34,36, $38,39,41,43,44,46,47,54,57-66]$, others followed up their participants for different periods, ranging from two months [22] to three $[27,37,40,42,48-50,56]$, six [31, $33,35,45,53]$, twelve [24,55] and twenty-four months [28,52].

Interventions that included improving diagnostic procedures to help physicians distinguish bacterial from viral infections led to very positive results [29,41,61, $63,65]$.

\section{Interventions in hospital care professionals}

Whereas most interventions concentrated on physicians (Table 1), some included a multidisciplinary intervention targeting physicians and nurses $[68,70,76,79,92]$, patients [80], and in one case, solely pharmacists [83]. Some studies identified the patients targeted, with these being elderly in five instances [68-70,87,92,93] children in three $[72,76,82]$. Table 3 summarizes the studies retrieved containing interventions for improving antibiotic use in hospital care. The diseases targeted were as follows: pneumonia in four cases [68,75,80,93]; urinary infections in two [70,72]; urinary and upper respiratory tract infections in one [69]; pneumonia, meningitis and urinary infection in one study [85], and bronchitis, community acquired pneumonia and chronic obstructive pulmonary disease in other [96]. Of the thirty-one papers, $6(20 \%)$ studied the efficacy/effectiveness of one or more interventions versus no intervention, using a control group [70,71,73,77,83,86]. Naughton [68] compared two strategies, a multidisciplinary intervention in physicians and nurses, and a physician-only intervention in ten skilled nursing facilities randomized into two groups, and reported no statistically significant differences between the two groups. Most of the reported hospitalbased interventions coincided with the implementation of protocols or new computer systems, with the result that post-intervention were compared with pre-intervention outcomes without the use of control groups.

While some studies [67,73,76,83,87-89] used passive interventions, all the others used active interventions or passive and active simultaneously. Twenty-four papers (78\%) [67,69-74,77,79-92,95,96] reported positive results for all outcome measures; 6 papers (20\%) [3,75,76, $78,93,94$ reported some outcomes as positive and others as positive statistically non-significant; and Naughton reported negative results [68].

In contrast to primary care in which only three studies [24,26,32] analyzed clinical outcomes, in hospital care some studies $[67,69,70,72,74,81,84,86,96]$ compared outcomes pre- and post-intervention to assess whether a reduction in antibiotic use might cause clinical alterations, and no influences were observed, namely, to length of hospital stay, and mortality, morbidity and/or readmission rates.

Many of the hospital-care studies highlighted the important role of clinical pharmacists in drawing up and implementing guidelines and policies for antibiotic use in hospital settings $[3,67,69,74,75,77-80,82,85,86,91,96]$.

\section{Studies design}

While 25 papers (53\%) [21,24,26-28,30,35-38,40,42-44, $49,50,53,54,56-59,62,63,65]$ reported randomized controlled studies in the case of primary care, a far lower number, i.e., 8 (26\%) [68,70,73,74,77,83,86,92], reported this type of study in the case of hospital care, and only one of these included cross-contamination control. Cross-contamination can occur when the participants of different intervention or control groups have close working relationships and might share information about the intervention, and this is important because differences in the results between the intervention and the control group may be influenced by this factor. In some studies physicians participated on a voluntary basis (they were invited to participate in the study), and their prescribing habits recorded during the intervention may not represent their real use of antibiotics [24,26-30,33,37,50, 61-63,65,70].

There were many differences in the analytical approaches adopted by the different studies: while some compared the results of the intervention with the situation at baseline, and some compared the results between groups pre- and post-intervention, others focused exclusively on the position post-intervention. There were few studies that conducted a follow-up after the intervention 
Table 4 Review studies covering interventions to improve antibiotic use

\begin{tabular}{|c|c|c|c|c|c|c|}
\hline Author (year) & Title of study & Study objectives & Inclusion criteria & Methods & $\begin{array}{l}\text { Number of } \\
\text { studies included }\end{array}$ & Review period \\
\hline $\begin{array}{l}\text { van der Velden } \\
\text { (2012) [13] }\end{array}$ & $\begin{array}{l}\text { Effectiveness of physician- } \\
\text { targeted interventions to } \\
\text { improve antibiotic use for } \\
\text { respiratory tract infections }\end{array}$ & $\begin{array}{l}\text { To assess the effectiveness of } \\
\text { physician-targeted interventions aiming } \\
\text { to improve antibiotic prescribing for } \\
\text { respiratory tract infections in primary } \\
\text { care, and to identify intervention } \\
\text { features mostly contributing to } \\
\text { intervention success. }\end{array}$ & $\begin{array}{l}\text { Studies with an intervention primarily } \\
\text { targeted at physicians in a primary care } \\
\text { setting aiming to improve antibiotic } \\
\text { prescribing for RTIs, conducted in a } \\
\text { high-income country, presenting a } \\
\text { standardized outcome of (first choice) } \\
\text { prescription measured in defined daily } \\
\text { dosage, } \\
\text { prescription or rates. }\end{array}$ & $\begin{array}{l}\text { Systematic review of studies } \\
\text { published in MEDLINE, EMBASE, } \\
\text { and the Cochrane Library. } \\
\text { Quantitative analysis to assess the } \\
\text { association between effectiveness } \\
\text { rates and intervention features. }\end{array}$ & 58 & $\begin{array}{l}\text { January } 1990 \\
\text { through July } \\
2009\end{array}$ \\
\hline $\begin{array}{l}\text { Charani, E (2011) } \\
{[12]}\end{array}$ & $\begin{array}{l}\text { Behaviour Change Strategies to } \\
\text { Influence Antimicrobial } \\
\text { Prescribing in Acute Care: A } \\
\text { Systematic Review }\end{array}$ & $\begin{array}{l}\text { To assess the effectiveness of } \\
\text { antimicrobial prescribing interventions } \\
\text { that either alone or in combination, } \\
\text { aim to influence behaviors in acute } \\
\text { care. }\end{array}$ & $\begin{array}{l}\text { Effective Practice and Organization of } \\
\text { Care (EPOC) model was adapted to } \\
\text { include additional criteria for review of } \\
\text { uncontrolled studies. Studies were } \\
\text { included only if they were conducted } \\
\text { in countries defined as having a } \\
\text { developed health care system. }\end{array}$ & $\begin{array}{l}\text { Systematic review of studies } \\
\text { published in MEDLINE, Applied } \\
\text { Social Sciences Index and Abstracts } \\
\text { (ASSIA), Business Source Complete, } \\
\text { The Cochrane Library, PsycINFO, } \\
\text { and the Database of Abstracts of } \\
\text { Reviews of Effectiveness (DARE) } \\
\text { and Health Management } \\
\text { Information Consortium (HMIC) }\end{array}$ & 10 & $\begin{array}{l}\text { January } 1999 \\
\text { through April } \\
2011\end{array}$ \\
\hline $\begin{array}{l}\text { Tonkin-Crine, S } \\
\text { (2011) [97] }\end{array}$ & $\begin{array}{l}\text { Antibiotic prescribing for acute } \\
\text { respiratory tract infections in } \\
\text { primary care: a systematic } \\
\text { review and meta-ethnography. }\end{array}$ & $\begin{array}{l}\text { To evaluate general practitioners' } \\
\text { perceptions about antibiotic } \\
\text { prescribing, and interventions aimed at } \\
\text { prudent prescribing. }\end{array}$ & $\begin{array}{l}\text { Studies that used qualitative methods } \\
\text { and analysis. }\end{array}$ & $\begin{array}{l}\text { Meta-synthesis of qualitative } \\
\text { research examining GP attitudes } \\
\text { and experiences of antibiotic } \\
\text { prescribing, and interventions } \\
\text { aimed at more prudent prescribing } \\
\text { for ARTI. }\end{array}$ & 12 & 1950-May 2011 \\
\hline Kaki, R (2011) [11] & $\begin{array}{l}\text { Impact of antimicrobial } \\
\text { stewardship in critical care: a } \\
\text { systematic review. }\end{array}$ & $\begin{array}{l}\text { To evaluate the evidence for } \\
\text { antimicrobial stewardship interventions } \\
\text { in the critical care unit. }\end{array}$ & $\begin{array}{l}\text { Studies that evaluate the effectiveness } \\
\text { of application of any intervention to } \\
\text { improve antimicrobial utilization and } \\
\text { within an intensive care setting, using } \\
\text { a modified Cochrane Registry EPOC } \\
\text { Database inclusion criteria. }\end{array}$ & $\begin{array}{l}\text { Systematic review of studies } \\
\text { published in OVID MEDLINE, } \\
\text { Embase and Cochrane databases }\end{array}$ & 24 & $\begin{array}{l}\text { January } 1996 \\
\text { through } \\
\text { December } 2010\end{array}$ \\
\hline $\begin{array}{l}\text { Boonacker, CWB } \\
\text { (2010) [10] }\end{array}$ & $\begin{array}{l}\text { Interventions in health care } \\
\text { professionals to improve } \\
\text { treatment in children with } \\
\text { upper respiratory tract } \\
\text { infections. }\end{array}$ & $\begin{array}{l}\text { To analyze which strategies are used to } \\
\text { promote evidence-based interventions } \\
\text { in the management of children with } \\
\text { URTI and assess the related } \\
\text { effectiveness and costs. }\end{array}$ & $\begin{array}{l}\text { Randomized controlled trials, non- } \\
\text { randomized controlled trials and } \\
\text { controlled before/after studies using } \\
\text { implementation methods to change } \\
\text { health care professionals' attitudes to } \\
\text { the treatment of children with URTI } \\
\text { and investigate the effectiveness of } \\
\text { implementation strategies. }\end{array}$ & $\begin{array}{l}\text { Systematic review of studies } \\
\text { published in Pubmed, Embase and } \\
\text { Cochrane Central Register of } \\
\text { Controlled Trials. }\end{array}$ & 17 & $\begin{array}{l}\text { Last search, } \\
\text { February } 2009\end{array}$ \\
\hline $\begin{array}{l}\text { Steinman, MA } \\
\text { (2006) [9] }\end{array}$ & $\begin{array}{l}\text { Improving antibiotic selection. } \\
\text { A systematic review and } \\
\text { quantitative analysis of quality } \\
\text { improvement strategies. }\end{array}$ & $\begin{array}{l}\text { To assess which interventions are most } \\
\text { effective in improving the prescribing } \\
\text { of recommended antibiotics for acute } \\
\text { outpatient infections. }\end{array}$ & $\begin{array}{l}\text { Clinical trials with contemporaneous or } \\
\text { strict historical controls that reported } \\
\text { data on antibiotic selection in acute } \\
\text { outpatient infections }\end{array}$ & $\begin{array}{l}\text { Systematic review with quantitative } \\
\text { analysis of the EPOC Database, } \\
\text { supplemented by MEDLINE and } \\
\text { hand-searches }\end{array}$ & 24 & $\begin{array}{l}\text { Last search, } \\
\text { November } \\
2004\end{array}$ \\
\hline
\end{tabular}


had ended, and those which did reported that the majority of positive results observed in the post-intervention period were lost over time.

No studies were found in which the interventions had been designed on the basis of the attitudes and behavior responsible for antibiotic prescribing or dispensing habits, despite the fact that many authors contend that this knowledge contributes to the success of educational interventions in health professionals $[69,79,80]$. In some studies $[28,33,49,79]$, however, interventions addressed barriers facing the individual prescriber, particularly when it came to dealing with diagnostic uncertainty, and were tailored to: overcoming any identified barriers and enable general practitioners (GPs) to reflect on their own prescribing; helping decrease uncertainty about appropriate disease management and appropriate prescribing; facilitating more patient-centered care; and being beneficial to implementation in practice. One study [39] assessed the impact of interventions on the knowledge, beliefs, and decision-making of primary care physicians, and two others, used workshops and focus-group discussions to determine the possible motivating factors underlying observed prescribing practices $[49,62]$. The importance of interventions being acceptable to physicians was highlighted by a recent systematic review [97].

All the studies underlined the importance of appropriate use of antibiotics to prevent the problem of microbial resistance, and stated that the most important aim of interventions to improve antibiotic use was to reduce this important public health problem. Even so, only one primary-care [46] and eight hospital-care studies $[67,84,85,87,89-91,93]$ analyzed improvement in bacterial susceptibility during the intervention. While some studies reported the reduction in the cost of antibiotic use, only five studies analyzed the effectiveness of intervention in terms of the cost of the intervention versus the cost of reducing antibiotic use $[73,78,81,84,86,96]$.

We found only two studies that addressed interventions (undertaken in Thailand and Vietnam, respectively) $[42,44]$ specifically designed to improve pharmacists' to combat the dispensing of antibiotics without prescription, despite there were studies which established that the sale of antibiotics without a prescription are a reality in some European countries [98-102]. Although some of the studies reviewed -mainly those pertaining to hospital care- reported the important role played by pharmacists in developing interventions to be undertaken in physicians and implementing antibiotic treatment guidelines and protocols in hospital settings, there were few studies with interventions targeted at pharmacists. Some authors stressed the usefulness of including pharmacists in teams tasked with drawing up recommendations and making decisions about antibiotic use in certain countries $[54,96,103,104]$.
Results obtained by our search showed that the majority of published studies about educational interventions describe active and multifaceted interventions. This finding is in accordance with a number of systematic metaanalyses of randomized controlled trials to improve health care practice, which conclude that highly interactive learning methods, such as educational outreach visits [105] workshops [106,107], small discussion groups $[107,108]$, individualized training sessions $[107,108]$, practice-based interventions [19] and case-based learning [109], are the most effective strategies.

Some recent review papers on interventions to improve antibiotic prescribing [9-11,97] (Table 4) focus on a limited set of intervention targets, such as acute outpatient infections, and more specifically on clinical knowledge and decision-making processes [9], specific populations (children), specific diseases (upper respiratory tract infections) [10] or purpose-designed noneducational (stewardship) interventions in specific hospital divisions, such as critical care [11] and acute care [12]. One paper [97] reviewed studies that evaluated GPs' perceptions about antibiotic prescribing and interventions aimed at prudent prescribing. Our study only analyzed educational interventions but was more extensive, in that it included interventions aimed at physicians and/ or pharmacists in both primary-care and hospital settings, and focused on any disease with antibiotic prescribing for child, adult or geriatric patients. In contrast to Steinman [9], who made a quantitative analyses of quality-improvement strategies, our review, like those of Boonacker [10], Kaki [11] and Charani [12], was a qualitative analysis.

As in the case of any systematic review, ours suffers from the limitation of publication bias. The inclusion criteria allowed for the review to cover a wide range of studies with different designs, something hindered us in making comparisons and performing a meta-analysis. Identification of the design proved a complex task, and it is therefore possible that some study may have been misclassified as regards design, due to an incomplete description of the methodology used. In many cases, deficiencies in the design and description of the intervention and identification of the sample made tabulating the study characteristics difficult.

\section{Conclusions}

The results yielded by our search show that there are many more papers on educational interventions in physicians than pharmacists. Respiratory disorders were the disease targeted by most studies, mainly in primary care. Published studies varied widely in terms of study design, outcome measures, outcome period, and definition of sample. Most studies used active or a mix of active and passive interventions, and reported that active 
interventions were more effective. Notwithstanding these heterogeneity, it can be concluded from the above: first, that educational interventions to improve antibiotic use are essential; and second, that in many studies such interventions are active and multifaceted, some of them include both physicians and pharmacists, and were designed taking these health professionals' attitudes and knowledge into account, in order to focus on the barriers so identified.

\section{Competing interests}

The authors declare that they have no competing interests.

\section{Authors' contributions}

$F R, L B, M T H, A F$ - concept the study and participated actively in the design of the study. FR and SS - performed the bibliographic search. FR, ATR and SS - extract data from the studies. FR, LB, MTH, AF - interpreted and discuss the data. FR writes the manuscript. All authors read and approved the manuscript.

\section{Acknowledgements}

Authors wish to express their thanks to the Foundation for Science and Technology (Fundação para a Ciência e Tecnologia - FCT), grants [PTDC/ SAU-ESA/105530/2008] and [Pest-OE/EGE/UI4056/2014] from the Portuguese Ministry of Science and Education, and to Health Research Fund (Fondo de Investigación Sanitaria) grants [P1081239 and PI09/90609] from the Spanish Ministry of Health. The funders had no role in study design, data collection and analysis, decision to publish or preparation of the manuscript.

\section{Author details}

${ }^{1}$ Health Sciences Research Centre, University of Beira Interior (Centro de Investigação em Ciências da Saúde - CICS/UBI), Av. Infante D. Henrique, 6200-506 Covilhã, Portugal. ${ }^{2}$ Centre for Cell Biology, University of Aveiro (Centro de Biologia Celular - CBC/UA); Campus Universitário de Santiago, 3810-193 Aveiro, Portugal. ${ }^{3}$ Research Unit for Inland Development, Polytechnic of Guarda (Unidade de Investigação para o Desenvolvimento do Interior - UDI/IPG), Av. Dr. Francisco Sá Carneiro n50, 6300-559 Guarda, Portugal. ${ }^{4}$ CESPU, Instituto de Investigação e Formação Avançada em Ciências e Tecnologias da Saúde, Rua Central de Gandra, 1317, 4585-116 Gandra, PRD, Portugal. ${ }^{5}$ Consortium for Biomedical Research in Epidemiology \& Public Health (CIBER en Epidemiología y Salud Pública - CIBERESP), University of Santiago de Compostela, R/ de San Francisco, s/n 15782 Santiago de Compostela, Spain.

Received: 11 August 2014 Accepted: 8 December 2014 Published: 15 December 2014

\section{References}

1. Spellberg B, Powers JH, Brass EP, Miller LG, Edwards JE: Trends in antimicrobial drug development: implications for the future. Clinical Infectious Diseases 2004, 38(9):1279-1286.

2. ECDC EMA: Technical Report - The Bacterial Chalange: time to react. Stockholm: 2009. on line in http://www.ecdc.europa.eu/en/publications/ Publications/0909 TER_The Bacterial_Challenge_Time_to_React.pdf

3. Deuster S, Roten I, Muehlebach S: Implementation of treatment guidelines to support judicious use of antibiotic therapy. Journal of Clinical Pharmacy and Therapeutics 2010, 35(1):71-78.

4. Goossens H, Ferech M, Vander Stichele R, Elseviers M: Outpatient antibiotic use in Europe and association with resistance: a cross-national database study. The Lancet 2005, 365(9459):579-587.

5. van de Sande-Bruinsma N, Grundmann H, Verloo D, Tiemersma E, Monen J, Goossens $H$, Ferech M, System EARS: Antimicrobial drug use and resistance in Europe. Emerging infectious diseases 2008, 14(11):1722.

6. Grigoryan L, Burgerhof JGM, Degener JE, Deschepper R, Lundborg CS, Monnet DL, Scicluna EA, Birkin J, Haaijer-Ruskamp FM: Determinants of self-medication with antibiotics in Europe: the impact of beliefs, country wealth and the healthcare system. Journal of Antimicrobial Chemotherapy 2008, 61(5):1172-1179.
7. Grigoryan L, Burgerhof JGM, Haaijer-Ruskamp FM, Degener JE, Deschepper R, Monnet DL, Di Matteo A, Scicluna EA, Bara A-C, Lundborg CS, Barkin J, SAR group: Is self-medication with antibiotics in Europe driven by prescribed use? Journal of Antimicrobial Chemotherapy 2007, 59(1):152-156.

8. Deschepper R, Grigoryan L, Lundborg C, Hofstede G, Cohen J, Kelen G, Deliens L, Haaijer-Ruskamp F: Are cultural dimensions relevant for explaining cross-national differences in antibiotic use in Europe? BMC Health Services Research 2008, 8(1):123.

9. Steinman MA, Ranji SR, Shojania KG, Gonzales R: Improving Antibiotic Selection: A Systematic Review and Quantitative Analysis of Quality Improvement Strategies. Medical Care 2006, 44(7):617-628. 610.1097/1001. mlr.0000215846.0000225591.0000215822.

10. Boonacker CWB, Hoes AW, Dikhoff M-J, Schilder AGM, Rovers MM: Interventions in health care professionals to improve treatment in children with upper respiratory tract infections. International journal of pediatric otorhinolaryngology 2010, 74(10):1113-1121.

11. Kaki R, Elligsen M, Walker S, Simor A, Palmay L, Daneman N: Impact of antimicrobial stewardship in critical care: a systematic review. Journal of Antimicrobial Chemotherapy 2011, 66(6):1223-1230.

12. Charani E, Edwards R, Sevdalis N, Alexandrou B, Sibley E, Mullett D, Franklin BD, Holmes A: Behavior Change Strategies to Influence Antimicrobial Prescribing in Acute Care: A Systematic Review. Clinical Infectious Diseases 2011, 53(7):651-662.

13. van der Velden AW, Pijpers EJ, Kuyvenhoven MM, Tonkin-Crine SK, Little P, Verheij TJ: Effectiveness of physician-targeted interventions to improve antibiotic use for respiratory tract infections. The British journal of general practice: the journal of the Royal College of General Practitioners 2012, 62(605):e801-e807.

14. Ranji SR, Steinman MA, Shojania KG, Sundaram V, Lewis R, Arnold S, Gonzales R: Closing the quality gap: a critical analysis of quality improvement strategies (vol. 4: antibiotic prescribing behavior). 2006.

15. Arnold SR, Straus SE, Arnold S: Interventions to improve antibiotic prescribing practices in ambulatory care. Cochrane Database Syst Rev 2005, 4(4):CD003539.

16. Davey P, Brown E, Fenelon L, Finch R, Gould I, Hartman G, Holmes A, Ramsay C, Taylor E, Wilcox M: Interventions to improve antibiotic prescribing practices for hospital inpatients (Review). 2007.

17. Lopez-Vazquez P, Vazquez-Lago JM, Figueiras A: Misprescription of antibiotics in primary care: a critical systematic review of its determinants. Journal of Evaluation in Clinical Practice 2012, 18(2):473-484.

18. Figueiras A, Sastre I, Gestal-Otero JJ: Effectiveness of educational interventions on the improvement of drug prescription in primary care: a critical literature review. Journal of Evaluation in Clinical Practice 2001, 7(2):223-241.

19. Davis DA, Thomson M, Oxman AD, Haynes R: Changing physician performance: A systematic review of the effect of continuing medical education strategies. JAMA 1995, 274(9):700-705.

20. Dollman WB, LeBlanc VT, Stevens L, O'Connor PJ, Turnidge JD: A community-based intervention to reduce antibiotic use for upper respiratory tract infections in regional South Australia. Medical journal of Australia 2005, 182(12):617-620.

21. Hrisos S, Eccles M, Johnston M, Francis J, Kaner E, Steen N, Grimshaw J: An intervention modelling experiment to change GPs' intentions to implement evidence-based practice: using theory-based interventions to promote GP management of upper respiratory tract infection without prescribing antibiotics \#2. BMC Health Services Research 2008, 8(1):10.

22. Hennessy TW, Petersen KM, Bruden D, Parkinson AJ, Hurlburt D, Getty M Schwartz B, Butler JC: Changes in Antibiotic-Prescribing Practices and Carriage of Penicillin-Resistant Streptococcus pneumoniae: A Controlled Intervention Trial in Rural Alaska. Clinical Infectious Diseases 2002, 34(12):1543-1550.

23. Rubin MA, Bateman K, Alder S, Donnelly S, Stoddard GJ, Samore MH: A Multifaceted Intervention to Improve Antimicrobial Prescribing for Upper Respiratory Tract Infections in a Small Rural Community. Clinical Infectious Diseases 2005, 40(4):546-553.

24. Naughton C, Feely J, Bennett K: A RCT evaluating the effectiveness and cost-effectiveness of academic detailing versus postal prescribing feedback in changing GP antibiotic prescribing. Journal of Evaluation in Clinical Practice 2009, 15(5):807-812.

25. Chazan B, Turjeman RBZ, Frost Y, Besharat B, Tabenkin H, Stainberg A, Sakran W, Raz R: Antibiotic consumption successfully reduced by a community intervention program. IMAJ-RAMAT GAN- 2007, 9(1):16. 
26. Briel $M$, Christ-Crain $M$, Young J, Schuetz $P$, Huber $P$, Periat $P$, Bucher $H$, Muller B: Procalcitonin-guided antibiotic use versus a standard approach for acute respiratory tract infections in primary care: study protocol for a randomised controlled trial and baseline characteristics of participating general practitioners [ISRCTN73182671]. BMC Family Practice 2005, 6(1):34

27. Monette J, Miller MA, Monette M, Laurier C, Boivin J-F, Sourial N, Le Cruguel J-P, Vandal A, Cotton-Montpetit M: Effect of an Educational Intervention on Optimizing Antibiotic Prescribing in Long-Term Care Facilities. Journal of the American Geriatrics Society 2007, 55(8):1231-1235.

28. Enriquez-Puga A, Baker R, Paul S, Villoro-Valdes R: Effect of educational outreach on general practice prescribing of antibiotics and antidepressants: A two-year randomised controlled trial. Scandinavian Journal of Primary Health Care 2009, 27(4):195-201.

29. Bjerrum L, Cots J, Llor C, Molist N, Munck A: Effect of intervention promoting a reduction in antibiotic prescribing by improvement of diagnostic procedures: a prospective, before and after study in general practice. Eur J Clin Pharmacol 2006, 62(11):913-918.

30. Mcisaac WJ, Goel V, To T, Permaul JA, Low DE: Effect on antibiotic prescribing of repeated clinical prompts to use a sore throat score. Journal of family practice 2002, 51(4):339-344.

31. Wheeler JG, Fair M, Simpson PM, Rowlands LA, Aitken ME, Jacobs RF: Impact of a Waiting Room Videotape Message on Parent Attitudes Toward Pediatric Antibiotic Use. Pediatrics 2001, 108(3):591-596.

32. Juzych NS, Banerjee M, Essenmacher L, Lerner S: Improvements in antimicrobial prescribing for treatment of upper respiratory tract infections through provider education. J GEN INTERN MED 2005, 20(10):901-905.

33. Smeets HM, Kuyvenhoven MM, Akkerman AE, Welschen I, Schouten GP, van Essen GA, Verheij TJM: Intervention with educational outreach at large scale to reduce antibiotics for respiratory tract infections: a controlled before and after study. Family Practice 2009, 26(3):183-187.

34. Mandryk JA, Mackson JM, Horn FE, Wutzke SE, Badcock C-A, Hyndman RJ, Weekes LM: Measuring change in prescription drug utilization in Australia. Pharmacoepidemiology and Drug Safety 2006, 15(7):477-484.

35. Stille CJ, Rifas-Shiman SL, Kleinman K, Kotch JB, Finkelstein JA: Physician Responses to a Community-Level Trial Promoting Judicious Antibiotic Use. The Annals of Family Medicine 2008, 6(3):206-212.

36. Finkelstein JA, Davis RL, Dowell SF, Metlay JP, Soumerai SB, Rifas-Shiman SL, Higham M, Miller Z, Miroshnik I, Pedan A, Platt R: Reducing Antibiotic Use in Children: A Randomized Trial in 12 Practices. Pediatrics 2001, 108(1):1-7.

37. Altiner A, Brockmann S, Sielk M, Wilm S, Wegscheider K, Abholz H-H: Reducing antibiotic prescriptions for acute cough by motivating GPs to change their attitudes to communication and empowering patients: a cluster-randomized intervention study. Journal of Antimicrobial Chemotherapy 2007, 60(3):638-644.

38. Légaré F, Labrecque M, LeBlanc A, Njoya M, Laurier C, Côté L, Godin G, Thivierge RL, O'Connor A, St-Jacques S: Training family physicians in shared decision making for the use of antibiotics for acute respiratory infections: a pilot clustered randomized controlled trial. Health Expectations 2011, 14:96-110.

39. Kiang KM, Kieke BA, Como-Sabetti K, Lynfield R, Besser RE, Belongia EA Clinician knowledge and beliefs after statewide program to promote appropriate antimicrobial drug use. Emerg Infect Dis 2005, 11(6):904-911.

40. Mohagheghi MA, Mosavi-Jarrahi A, Khatemi-Moghaddam M, Afhami S, Khodai S, Azemoodeh O: Community-based outpatient practice of antibiotics use in Tehran. Pharmacoepidemiology and Drug Safety 2005, 14(2):135-138

41. Irurzun C, González M, Recondo M, Urtasun M: Efectividad de la aplicación de un protocolo clínico para el manejo de la faringitis aguda en adultos. TITLEREVISTA 2005, 35(01):22-29.

42. Chalker J, Ratanawijitrasin S, Chuc NTK, Petzold M, Tomson G: Effectiveness of a multi-component intervention on dispensing practices at private pharmacies in Vietnam and Thailand-a randomized controlled trial. Social Science \& Medicine 2005, 60(1):131-141.

43. Finkelstein JA, Huang SS, Kleinman K, Rifas-Shiman SL, Stille CJ, Daniel J, Schiff N, Steingard R, Soumerai SB, Ross-Degnan D, Goldman D, Platt R: Impact of a 16-Community Trial to Promote Judicious Antibiotic Use in Massachusetts. Pediatrics 2008, 121(1):e15-e23.

44. Chuc NTK, Larsson M, Do NT, Diwan VK, Tomson GB, Falkenberg T: Improving private pharmacy practice: A multi-intervention experiment in Hanoi, Vietnam. Journal of clinical epidemiology 2002, 55(11):1148-1155.
45. Belongia EA, Sullivan BJ, Chyou P-H, Madagame E, Reed KD, Schwartz B: A Community Intervention Trial to Promote Judicious Antibiotic Use and Reduce Penicillin-Resistant Streptococcus pneumoniae Carriage in Children. Pediatrics 2001, 108(3):575-583.

46. Belongia EA, Knobloch MJ, Kieke BA, Davis JP, Janette C, Besser RE: Impact of statewide program to promote appropriate antimicrobial drug use. Emerg Infect Dis 2005, 11(6):912-920.

47. Greene RA, Beckman H, Chamberlain J, Partridge G, Miller M, Burden D, Kerr $\mathrm{J}$ : Increasing adherence to a community-based guideline for acute sinusitis through education, physician profiling, and financial incentives. The American journal of managed care 2004, 10(10):670-678.

48. Teng $\mathrm{CL}$, Achike Fl, Phua KL, Nurjahan Ml, Mastura I, Asiah HN, Mariam AM, Narayanan S, Norsiah A, Sabariah I: Modifying antibiotic prescribing: the effectiveness of academic detailing plus information leaflet in a Malaysian primary care setting. The Medical journal of Malaysia 2006, 61(3):323-331

49. Awad Al, Eltayeb IB, Baraka OZ: Changing antibiotics prescribing practices in health centers of Khartoum State. Sudan. Eur J Clin Pharmacol 2006, 62(2):135-142

50. Welschen I, Kuyvenhoven MM, Hoes AW, Verheij TJM: Effectiveness of a multiple intervention to reduce antibiotic prescribing for respiratory tract symptoms in primary care: randomised controlled trial. BMJ 2004. 329:431

51. Gonzales R, Sauaia A, Corbett KK, Maselli JH, Erbacher K, Leeman-castillo BA, Darr CA, Houck PM: Antibiotic Treatment of Acute Respiratory Tract Infections in the Elderly: Effect of a Multidimensional Educational Intervention. Journal of the American Geriatrics Society 2004, 52(1):39-45.

52. Colomina Rodríguez J, Domínguez Márquez V, Gimeno Vilarrasa F, Sarrió Montes G, Guerrero Espejo A: Impacto de un modelo integrado para el uso racional de antimicrobianos (Proyecto Miura) en un área de salud. Revista Española de Salud Pública 2010, 84:281-291.

53. Hickman DE, Stebbins MR, Hanak JR, Guglielmo BJ: Pharmacy-Based Intervention to Reduce Antibiotic Use for Acute Bronchitis. Annals of Pharmacotherapy 2003, 37(2):187-191.

54. Coenen S, Van Royen P, Michiels B, Denekens J: Optimizing antibiotic prescribing for acute cough in general practice: a cluster-randomized controlled trial. Journal of Antimicrobial Chemotherapy 2004, 54(3):661-672.

55. Perz JF, Craig AS, Coffey CS, Jorgensen DM, Mitchel E, Hall S, Schaffner W, Griffin MR: CHanges in antibiotic prescribing for children after a communitywide campaign. JAMA 2002, 287(23):3103-3109.

56. Søndergaard J, Andersen M, Støvring H, Kragstrup J: Mailed prescriber feedback in addition to a clinical guideline has no impact: a randomised, controlled trial. Scandinavian Journal of Primary Health Care 2003, 21(1):47-51.

57. Doyne EO, Alfaro M, Siegel RM, Atherton HD, Schoettker PJ, Bernier J, Kotagal UR: A randomized controlled trial to change antibiotic prescribing patterns in a community. Archives of Pediatrics \& Adolescent Medicine 2004, 158(6):577-583.

58. Bauchner H, Marchant CD, Bisbee A, Heeren T, Wang B, McCabe M, Pelton S, Group B-BPR: Effectiveness of Centers for Disease Control and Prevention Recommendations for Outcomes of Acute Otitis Media. Pediatrics 2006, 117(4):1009-1017.

59. Christakis DA, Zimmerman FJ, Wright JA, Garrison MM, Rivara FP, Davis RL: A Randomized Controlled Trial of Point-of-Care Evidence to Improve the Antibiotic Prescribing Practices for Otitis Media in Children. Pediatrics 2001, 107(2):e15.

60. Småbrekke L, Berild D, Giaever A, Myrbakk T, Fuskevåg A, Ericson JU, Flaegstad T, Olsvik $\varnothing$, Ringertz SH: Educational Intervention for Parents and Healthcare Providers Leads to Reduced Antibiotic Use in Acute Otitis Media. Scandinavian Journal of Infectious Diseases 2002, 34(9):657-659.

61. Bjerrum L, Munck A, Gahrn-Hansen B, Hansen M, Jarbol D, Cordoba G, Llor C, Cots J, Hernandez S, Lopez-Valcarcel B, Perez A, Caballero L, von der Heyde W, Radzevicience R, Jurgutis A, Reutskiy A, Egorova E, Strandberg EL, Ovhed I, Molstad S, Stichele RV, Benko R, Vlahovic-Palcevski V, Lionis C, Ronning M: Health Alliance for prudent antibiotic prescribing in patients with respiratory tract infections (HAPPY AUDIT) -impact of a non-randomised multifaceted intervention programme. BMC Family Practice 2011, 12(1):52

62. Regev-Yochay G, Raz M, Dagan R, Roizin H, Morag B, Hetman S, Ringel S, Ben-Israel N, Varon M, Somekh E, Rubinstein E: Reduction in Antibiotic Use 
Following a Cluster Randomized Controlled Multifaceted Intervention: The Israeli Judicious Antibiotic Prescription Study. Clinical Infectious Diseases 2011, 53(1):33-41.

63. Llor C, Cots JM, González López-Valcárcel B, Alcántara JD, García G, Arranz J, Monedero MJ, Ortega J, Pineda V, Guerra G, Gómez M, Hernández S, Paredes J, Cid M, Pérez C: Effect of two interventions on reducing antibiotic prescription in pharyngitis in primary care. Journal of Antimicrobial Chemotherapy 2011, 66(1):210-215.

64. Weiss K, Blais R, Fortin A, Lantin S, Gaudet M: Impact of a Multipronged Education Strategy on Antibiotic Prescribing in Quebec. Canada. Clinical Infectious Diseases 2011, 53(5):433-439.

65. Llor C, Madurell J, Balagué-Corbella M, Gómez M, Cots JM: Impact on antibiotic prescription of rapid antigen detection testing in acute pharyngitis in adults: a randomised clinical trial. British Journal of General Practice 2011, 61(586):e244-e251.

66. McKay RM, Vrbova L, Fuertes E, Chong M, David S, Dreher K, Purych D, Blondel-Hill E, Henry B, Marra F, Kendall PR, Patrick DM: Evaluation of the Do Bugs Need Drugs? program in British Columbia: Can we curb antibiotic prescribing? Canadian Journal of Infectious Diseases \& Medical Microbiology 2011, 22(1):19-24.

67. Chang MT, Wu TH, Wang CY, Jang TN, Huang CY: The impact of an intensive antimicrobial control program in a Taiwanese medical center. Pharm World Sci 2006, 28(4):257-264.

68. Naughton BJ, Mylotte JM, Ramadan F, Karuza J, Priore RL: Antibiotic Use, Hospital Admissions, and Mortality Before and After Implementing Guidelines for Nursing Home-Acquired Pneumonia. Journal of the American Geriatrics Society 2001, 49(8):1020-1024.

69. Lutters M, Harbarth S, Janssens J-P, Freudiger H, Herrmann F, Michel J-P, Vogt N: Effect of a Comprehensive, Multidisciplinary, Educational Program on the Use of Antibiotics in a Geriatric University Hospital. Journal of the American Geriatrics Society 2004, 52(1):112-116.

70. Loeb M, Brazil K, Lohfeld L, McGeer A, Simor A, Stevenson K, Zoutman D, Smith S, Liu X, Walter SD: Effect of a multifaceted intervention on number of antimicrobial prescriptions for suspected urinary tract infections in residents of nursing homes: cluster randomised controlled trial. BMJ 2005, 331(7518):669.

71. Lesprit P, Duong T, Girou E, Hemery F, Brun-Buisson C: Impact of a computer-generated alert system prompting review of antibiotic use in hospitals. Journal of Antimicrobial Chemotherapy 2009, 63(5):1058-1063.

72. Akter SFU, Heller RD, Smith AJ, Milly AF: Impact of a training intervention on use of antimicrobials in teaching hospitals. The Journal of Infection in Developing Countries 2009, 3(06):447-451.

73. Paul M, Andreassen S, Tacconelli E, Nielsen AD, Almanasreh N, Frank U, Cauda R, Leibovici L, Group obotTS: Improving empirical antibiotic treatment using TREAT, a computerized decision support system: cluster randomized trial. Journal of Antimicrobial Chemotherapy 2006, 58(6):1238-1245.

74. Camins BC, King MD, Wells JB, Googe HL, Patel M, Kourbatova EV, Blumberg HM: Impact of an Antimicrobial Utilization Program on Antimicrobial Use at a Large Teaching Hospital: A Randomized Controlled Trial •. Infection Control and Hospital Epidemiology 2009, 30(10):931-938.

75. Westphal JF, Jehl F, Javelot H, Nonnenmacher C: Enhanced physician adherence to antibiotic use guidelines through increased availability of guidelines at the time of drug ordering in hospital setting. Pharmacoepidemiology and Drug Safety 2011, 20(2):162-168.

76. Mullett CJ, Evans RS, Christenson JC, Dean JM: Development and Impact of a Computerized Pediatric Antiinfective Decision Support Program. Pediatrics 2001, 108(4):e75.

77. von Gunten V, Reymond J-P, Beney J: Clinical and economic outcomes of pharmaceutical services related to antibiotic use: a literature review. Pharmacy World \& Science 2007, 29(3):146-163.

78. Ansari F, Gray K, Nathwani D, Phillips G, Ogston S, Ramsay C, Davey P. Outcomes of an intervention to improve hospital antibiotic prescribing: interrupted time series with segmented regression analysis. Journal of Antimicrobial Chemotherapy 2003, 52(5):842-848.

79. Kisuule F, Wright S, Barreto J, Zenilman J: Improving antibiotic utilization among hospitalists: A pilot academic detailing project with a public health approach. Journal of Hospital Medicine 2008, 3(1):64-70.

80. Halm EA, Horowitz C, Silver A, Fein A, Dlugacz YD, Hirsch B, Chassin MR: Llmited impact of a multicenter intervention to improve the quality and efficiency of pneumonia care*. CHEST Journal 2004, 126(1):100-107.
81. López-Medrano F, San Juan R, Serrano O, Chaves F, Lumbreras C, Lizasoaín M, Herreros de Tejada A, Aguado JM: PACTA: efecto de un programa no impositivo de control y asesoramiento del tratamiento antibiótico sobre la disminución de los costes y el descenso de ciertas infecciones nosocomiales. TITLEREVISTA 2005, 23(04):186-190.

82. Agwu AL, Lee CKK, Jain SK, Murray KL, Topolski J, Miller RE, Townsend T, Lehmann CU: A World Wide Web-Based Antimicrobial Stewardship Program Improves Efficiency, Communication, and User Satisfaction and Reduces Cost in a Tertiary Care Pediatric Medical Center. Clinical Infectious Diseases 2008, 47(6):747-753.

83. Barenfanger J, Short MA, Groesch AA: Improved Antimicrobial Interventions Have Benefits. Journal of Clinical Microbiology 2001, 39(8):2823-2828.

84. Rüttimann S, Keck B, Hartmeier C, Maetzel A, Bucher HC: Long-Term Antibiotic Cost Savings from a Comprehensive Intervention Program in a Medical Department of a University-Affiliated Teaching Hospital. Clinical Infectious Diseases 2004, 38(3):348-356.

85. Martin C, Ofotokun I, Rapp R, Empey K, Armitstead J, Pomeroy C, Hoven A, Evans M: Results of an antimicrobial control program at a university hospital. American Journal of Health-System Pharmacy 2005, 62(7):732-738.

86. Solomon DH, Van Houten L, Glynn RJ, Baden L, Curtis K, Schrager H, Avorn J: Academic detailing to improve use of broad-spectrum antibiotics at an academic medical center. Archives of Internal Medicine 2001, 161(15):1897-1902.

87. Fowler S, Webber A, Cooper BS, Phimister A, Price K, Carter Y, Kibbler CC, Simpson AJH, Stone SP: Successful use of feedback to improve antibiotic prescribing and reduce Clostridium difficile infection: a controlled interrupted time series. Journal of Antimicrobial Chemotherapy 2007 59(5):990-995.

88. Sintchenko V, Iredell JR, Gilbert GL, Coiera E: Handheld Computer-based Decision Support Reduces Patient Length of Stay and Antibiotic Prescribing in Critical Care. Journal of the American Medical Informatics Association 2005, 12(4):398-402

89. Yong MK, Buising KL, Cheng AC, Thursky KA: Improved susceptibility of Gram-negative bacteria in an intensive care unit following implementation of a computerized antibiotic decision support system. Journal of Antimicrobial Chemotherapy 2010, 65(5):1062-1069.

90. Meyer E, Schwab F, Pollitt A, Bettolo W, Schroeren-Boersch B, Trautmann M: Impact of a Change in Antibiotic Prophylaxis on Total Antibiotic Use in a Surgical Intensive Care Unit. Infection 2010, 38(1):19-24.

91. Thursky KA, Buising KL, Bak N, Macgregor L, Street AC, Macintyre CR, Presneill JJ, Cade JF, Brown GV: Reduction of broad-spectrum antibiotic use with computerized decision support in an intensive care unit. International Journal for Quality in Health Care 2006, 18(3):224-231.

92. Pettersson E, Vernby $\AA$, Mölstad S, Lundborg CS: Can a multifaceted educational intervention targeting both nurses and physicians change the prescribing of antibiotics to nursing home residents? A cluster randomized controlled trial. Journal of Antimicrobial Chemotherapy 2011, 66(11):2659-2666.

93. Tängdén T, Eriksson B-M, Melhus Å, Svennblad B, Cars O: Radical reduction of cephalosporin use at a tertiary hospital after educational antibiotic intervention during an outbreak of extended-spectrum $\beta$-lactamaseproducing Klebsiella pneumoniae. Journal of Antimicrobial Chemotherapy 2011, 66(5):1161-1167.

94. Talpaert MJ, Gopal Rao G, Cooper BS, Wade P: Impact of guidelines and enhanced antibiotic stewardship on reducing broad-spectrum antibiotic usage and its effect on incidence of Clostridium difficile infection. Journal of Antimicrobial Chemotherapy 2011, 66(9):2168-2174.

95. Bevilacqua S, Demoré B, Erpelding M-L, Boschetti E, May T, May I, Rabaud C, Thilly N: Effects of an operational multidisciplinary team on hospital antibiotic use and cost in France: a cluster controlled trial. Int J Clin Pharm 2011, 33(3):521-528.

96. Shen J, Sun Q, Zhou X, Wei Y, Qi Y, Zhu J, Yan T: Pharmacist interventions on antibiotic use in inpatients with respiratory tract infections in a Chinese hospital. Int J Clin Pharm 2011, 33(6):929-933.

97. Tonkin-Crine S, Yardley L, Little P: Antibiotic prescribing for acute respiratory tract infections in primary care: a systematic review and meta-ethnography. Journal of Antimicrobial Chemotherapy 2011, 66(10):2215-2223.

98. Llor C, Cots JM: The Sale of Antibiotics without Prescription in Pharmacies in Catalonia. Spain. Clinical Infectious Diseases 2009 48(10):1345-1349. 
99. Apisarnthanarak A, Mundy LM: Comparison of Methods of Measuring Pharmacy Sales of Antibiotics without Prescriptions in Pratumthani, Thailand. Infection Control and Hospital Epidemiology 2009, 30(11):1130-1132.

100. Plachouras D, Kavatha D, Antoniadou A, Giannitsioti E, Poulakou G, Kanellakopoulou K, Giamarellou H: Dispensing of antibiotics without prescription in Greece, 2008: another link in the antibiotic resistance chain. Euro Surveill 2010, 15(7):19488.

101. Llor C, Monnet D, Cots J: Small pharmacies are more likely to dispense antibiotics without a medical prescription than large pharmacies in Catalonia. Spain. Euro Surveill 2010, 15:32.

102. Volpato DE, Souza BV, Dalla Rosa LG, Melo LH, Daudt CAS, Deboni L: Use of antibiotics without medical prescription. Brazilian Journal of Infectious Diseases 2005, 9:288-291.

103. Cars O, Mölstad S, Melander A: Variation in antibiotic use in the European Union. The Lancet 2001, 357(9271):1851-1853.

104. Gross R, Morgan AS, Kinky DE, Weiner M, Gibson GA, Fishman NO: Impact of a Hospital-Based Antimicrobial Management Program on Clinical and Economic Outcomes. Clinical Infectious Diseases 2001, 33(3):289-295.

105. O'Brien MA, Rogers S, Jamtvedt G, Oxman AD, Odgaard-Jensen J, Kristoffersen DT, Forsetlund L, Bainbridge D, Freemantle N, Davis D: Educational outreach visits: effects on professional practice and health care outcomes. Cochrane database syst rev 2007, 4(4)CD000172.

106. Forsetlund L, Bjorndal A, Rashidian A, Jamtvedt G, O'Brien MA, Wolf F, Davis D, Odgaard-Jensen J, Oxman AD: Continuing education meetings and workshops: effects on professional practice and health care outcomes. Cochrane Database Syst Rev 2009, 2, CD003030.

107. Davis D, O'Brien M, Freemantle N, Wolf FM, Mazmanian P, Taylor-Vaisey A: Impact of formal continuing medical education: Do conferences, workshops, rounds, and other traditional continuing education activities change physician behavior or health care outcomes? JAMA 1999, 282(9):867-874.

108. Figueiras A, Sastre I, Tato F, Rodríguez C, Lado E, Caamaño F, Gestal-Otero Jj: One-to-One Versus Group Sessions to Improve Prescription in Primary Care: A Pragmatic Randomized Controlled Trial. Medical Care 2001, 39(2):158-167.

109. Ribeiro-Vaz I, Herdeiro MT, Polónia J, Figueiras A: Estratégias para aumentar a sensibilidade da farmacovigilância em Portugal. Revista de Saúde Pública 2011, 45:129-135.

doi:10.1186/1471-2458-14-1276

Cite this article as: Roque et al.: Educational interventions to improve prescription and dispensing of antibiotics: a systematic review. BMC Public Health 2014 14:1276.

\section{Submit your next manuscript to BioMed Central and take full advantage of:}

- Convenient online submission

- Thorough peer review

- No space constraints or color figure charges

- Immediate publication on acceptance

- Inclusion in PubMed, CAS, Scopus and Google Scholar

- Research which is freely available for redistribution 